\title{
One Decade of Online Patient Feedback: Longitudinal Analysis of Data From a German Physician Rating Website
}

\author{
Martin Emmert ${ }^{1 *}$, MHMM, PhD; Stuart McLennan ${ }^{2,3^{*}}$, MBHL, PhD \\ ${ }^{1}$ Institute for Healthcare Management \& Health Sciences, University of Bayreuth, Bayreuth, Germany \\ ${ }^{2}$ Institute of History and Ethics in Medicine, Technical University of Munich, Munich, Germany \\ ${ }^{3}$ Institute for Biomedical Ethics, University of Basel, Basel, Switzerland \\ *all authors contributed equally
}

\section{Corresponding Author:}

Martin Emmert, MHMM, PhD

Institute for Healthcare Management \& Health Sciences

University of Bayreuth

Prieserstraße 2

Bayreuth, 95444

Germany

Phone: 4992155 ext 4827

Email: martin.emmert@uni-bayreuth.de

\begin{abstract}
Background: Feedback from patients is an essential element of a patient-oriented health care system. Physician rating websites (PRWs) are a key way patients can provide feedback online. This study analyzes an entire decade of online ratings for all medical specialties on a German PRW.
\end{abstract}

Objective: The aim of this study was to examine how ratings posted on a German PRW have developed over the past decade. In particular, it aimed to explore (1) the distribution of ratings according to time-related aspects (year, month, day of the week, and hour of the day) between 2010 and 2019, (2) the number of physicians with ratings, (3) the average number of ratings per physician, (4) the average rating, (5) whether differences exist between medical specialties, and (6) the characteristics of the patients rating physicians.

Methods: All scaled-survey online ratings that were posted on the German PRW jameda between 2010 and 2019 were obtained.

Results: In total, 1,906,146 ratings were posted on jameda between 2010 and 2019 for 127,921 physicians. The number of rated physicians increased constantly from 19,305 in 2010 to 82,511 in 2018 . The average number of ratings per rated physicians increased from 1.65 (SD 1.56) in 2010 to 3.19 (SD 4.69) in 2019. Overall, $75.2 \%(1,432,624 / 1,906,146)$ of all ratings were in the best rating category of "very good," and 5.7\% $(107,912 / 1,906,146)$ of the ratings were in the lowest category of "insufficient." However, the mean of all ratings was 1.76 (SD 1.53) on the German school grade 6-point rating scale (1 being the best) with a relatively constant distribution over time. General practitioners, internists, and gynecologists received the highest number of ratings $(343,242,266,899$, and 232,914, respectively). Male patients, those of higher age, and those covered by private health insurance gave significantly $(P<.001)$ more favorable evaluations compared to their counterparts. Physicians with a lower number of ratings tended to receive ratings across the rating scale, while physicians with a higher number of ratings tended to have better ratings. Physicians with between 21 and 50 online ratings received the lowest ratings (mean 1.95, SD 0.84), while physicians with >100 ratings received the best ratings (mean 1.34, SD 0.47).

Conclusions: This study is one of the most comprehensive analyses of PRW ratings to date. More than half of all German physicians have been rated on jameda each year since 2016, and the overall average number of ratings per rated physicians nearly doubled over the decade. Nevertheless, we could also observe a decline in the number of ratings over the last 2 years. Future studies should investigate the most recent development in the number of ratings on both other German and international PRWs as well as reasons for the heterogeneity in online ratings by medical specialty.

(J Med Internet Res 2021;23(7):e24229) doi: $\underline{10.2196 / 24229}$

\section{KEYWORDS}

physician rating websites; patient satisfaction; patient feedback; online ratings 


\section{Introduction}

Feedback from patients is an essential element of a patient-oriented health care system [1]. Patients' views and opinions on the care they have experienced can help health care organizations and professionals identify areas that need to be improved and can also help other patients with decision making when choosing where to receive health care [2]. Health care organizations and professionals can gather patient feedback in a variety of ways, including by conducting patient surveys, audits, interviews, focus groups, and deliberative events [3]. Patients have also always been able to actively share their views and opinions about the care they received with family and friends or with health care organizations and professionals via unsolicited comments or complaints. However, patients increasingly also have the ability to share their views and opinions on the internet and social media [4-7].

Physician rating websites (PRWs) are one of the key opportunities for patients to provide feedback online [4,7]. A systematic search of PRWs in 2018 identified 143 websites from 12 countries; however, the majority of websites were commercially operated in the United States and Germany [8]. Previous research involving PRW ratings in Germany and other countries has highlighted some common themes, including incomplete lists of physicians, a low number of physicians rated, a low number of ratings per physician that are overwhelmingly positive, and unstructured and different rating systems, which has raised concerns about the representativeness, validity, and usefulness of feedback on PRWs [7,9-30]. Medical associations have also often expressed strong opposition to PRWs, concerned that they will be used for doctorbashing or defamation $[31,32]$. Countries have different legal frameworks with regards to data protection, and previous research suggests that restrictive legal environments (eg, Switzerland) may be having an impact of the types of ratings on PRWs [28,29]. However, the legal basis for PRWs in Germany is reasonably liberal and well established. The Federal Court of Justice of Germany confirmed in 2014 the permissibility of ratings on the basis of the right to freedom of expression and that the anonymity of raters can only be lifted in exceptional cases $[33,34]$. Research also indicates that PRWs in Germany are having some success in influencing patient decision making and quality improvement $[17,35]$.

However, most studies examining PRWs ratings have typically focused on a certain year (eg, $[13,18,21])$, a certain medical specialty (eg, [22,23,36-40]), certain cities or regions (eg, $[14,26,41])$, or with a (more or less) randomly selected sample of physicians or ratings (eg, [14,21,26,36,41]). There is therefore a need for a more comprehensive examination of PRW ratings, to reveal a more generalizable view of ratings and allow trends in rating habits to be identified. As far as we are aware, only 2 studies from the United States [13] and Canada [27] have presented such findings.

This study takes a different approach from most previous studies and analyzes an entire decade of online ratings for all medical specialties on the German PRW, jameda [14,21,26,42] (Please note that the data are not publicly available but may be provided from the provider of the website for research purposes upon request.). Jameda was founded in 2007 and since 2016, has been a wholly owned subsidiary of Burda Digital GmbH. The commercial website provides users with a categorized search function to find suitable physicians, the ability to make appointments with physicians online, the possibility to have video consultations with physicians, an encyclopedia with information from experts on health topics, and an opportunity to rate physicians on a predefined grading system or leave narrative comments. In Germany, a total of 25 PRWs have been identified [8]; however, previous research has indicated that jameda is the German PRW with the highest public awareness, usage, and number of ratings given $[4,14,26]$.

The aim of this study was to examine how ratings posted on the German PRW jameda have developed over the past decade. In particular, it aimed to explore (1) the distribution of ratings according to time-related aspects (year, month, day of the week, and hour of the day) between 2010 and 2019, (2) the number of physicians with ratings, (3) the average number of ratings per physician, (4) the average rating, (5) whether differences exist between medical specialties, and (6) the characteristics of the patients rating physicians.

\section{Methods}

\section{Overview}

All scaled-survey online ratings that were posted on jameda between 2010 and 2019 were provided by jameda. Ratings on jameda are given according to the 6-point grading system used in German schools (1=very good, 2=good, 3=satisfactory, 4=fair, $5=$ deficient, and $6=$ insufficient) [24], in relation to 5 questions: (1) satisfaction with the treatment provided by the physician, (2) the physician's explanation about the illness and treatment, (3) the relationship of trust with the physician, (4) the time the physician spent with the patient, and (5) friendliness of the physician. Additionally, a mean score ("overall performance") is calculated based on the results for Q1 to Q5 [24]. The data also contained the physician's year of birth and medical specialty, as well as the rating patient's gender, age, and health insurance status.

\section{Statistical Analysis}

Descriptive statistics included means and SDs for continuous variables as well as numbers and percentages for categorical variables. To analyze whether differences existed between 2 groups, the Mann-Whitney $U$ test was used for continuous nonparametric variables, and the Kruskal-Wallis test was applied to determine differences between more than 2 groups. The Shapiro-Wilk test was used to examine the normality of the data distribution. Cohen $d$ was calculated to measure the magnitude of the effect size by comparing the standardized difference between the means of 2 groups. All statistical analyses were conducted using SPSS version 22.0 (IBM Corp, Armonk, NY). Differences were considered to be significant if $P<.05$ and highly significant if $P<.001$. 


\section{Results}

\section{Distribution of Ratings and Mean Ratings}

In total, 1,906,146 ratings were posted on jameda between 2010 and 2019 (see Table 1). The highest proportions of ratings were left in $2017(293,744 / 1,906,146,15.41 \%)$ and 2018 $(292,721 / 1,906,146,15.36 \%)$. In 2019 , there was a decline in the number of ratings $(232,739 / 1,906,146,12.21 \%)$ in comparison with the previous years. Ratings were distributed throughout the months of the year relatively equally (minimum in December: 143,620/1,906,146, 7.53\%; maximum in March: $173,865 / 1,906,146,9.12 \%)$, but more variation was found by day of the week (minimum on Saturdays: 123,024/1,906,146,
6.45\%; maximum on Tuesdays: 356,128/1,906,146, 18.68\%) and by hour of the day (minimum during 3-4 am: 4659/1,906,146, 0.24\%; maximum during 11-12 am: $152,606 / 1,906,146,8.00 \%)$. Likewise, the mean ratings were relatively similar across years (minimum in 2019: mean 1.71, SD 1.52; maximum in 2013: mean 1.83, SD 1.56), months (minimum in January: mean 1.73, SD 1.49; maximum in August: mean 1.77, SD 1.54), and days (minimum on Sunday: mean 1.68, SD 1.45; maximum on Monday: mean 1.78, SD 1.54). However, more variation could be seen by hour of the day (minimum during 7-8 am: mean 1.67, SD 1.43; maximum during 2-3 am and 3-4 am: mean 2.05, SD 1.75 and mean 2.05, SD 1.72 , respectively). 
Table 1. Distribution of ratings $(\mathrm{N}=1,906,146)$ and mean ratings.

\begin{tabular}{|c|c|c|}
\hline Timeframe & Ratings, n (\%) & Mean rating, mean (SD) \\
\hline \multicolumn{3}{|l|}{ Year } \\
\hline 2010 & $31,908(1.67)$ & $1.73(1.42)$ \\
\hline 2011 & $61,726(3.23)$ & $1.74(1.44)$ \\
\hline 2012 & $98,041(5.14)$ & $1.77(1.50)$ \\
\hline 2013 & $154,119(8.08)$ & $1.83(1.56)$ \\
\hline 2014 & $219,319(11.51)$ & $1.81(1.54)$ \\
\hline 2015 & $237,354(12.45)$ & $1.79(1.54)$ \\
\hline 2016 & $284,475(14.92)$ & $1.71(1.48)$ \\
\hline 2017 & $293,744(15.41)$ & $1.73(1.52)$ \\
\hline 2018 & $292,721(15.36)$ & $1.78(1.57)$ \\
\hline 2019 & $232,739(12.21)$ & $1.71(1.52)$ \\
\hline \multicolumn{3}{|l|}{ Month } \\
\hline January & $170,699(9.00)$ & $1.73(1.49)$ \\
\hline February & $167,728(8.80)$ & $1.77(1.53)$ \\
\hline March & $173,865(9.11)$ & $1.77(1.53)$ \\
\hline April & $151,098(7.93)$ & $1.77(1.53)$ \\
\hline May & $152,995(8.02)$ & $1.76(1.53)$ \\
\hline June & $147,422(7.73)$ & $1.76(1.53)$ \\
\hline July & $160,596(8.43)$ & $1.77(1.53)$ \\
\hline August & $151,544(7.95)$ & $1.77(1.54)$ \\
\hline September & $155,261(8.15)$ & $1.75(1.52)$ \\
\hline October & $161,630(8.48)$ & $1.77(1.53)$ \\
\hline November & $169,688(8.90)$ & $1.75(1.52)$ \\
\hline December & $143,620(7.53)$ & $1.73(1.51)$ \\
\hline \multicolumn{3}{|l|}{ Day of the week } \\
\hline Monday & $342,025(17.94)$ & $1.78(1.54)$ \\
\hline Tuesday & $356,128(18.68)$ & $1.78(1.54)$ \\
\hline Wednesday & $329,457(17.28)$ & $1.75(1.52)$ \\
\hline Thursday & $337,364(17.70)$ & $1.76(1.53)$ \\
\hline Friday & $267,234(14.02)$ & $1.77(1.54)$ \\
\hline Saturday & $123,024(6.45)$ & $1.74(1.52)$ \\
\hline Sunday & $150,914(7.91)$ & $1.68(1.45)$ \\
\hline \multicolumn{3}{|l|}{ Hour of the day } \\
\hline $0-1$ & $23,689(1.24)$ & $1.96(1.68)$ \\
\hline $1-2$ & $11,852(0.62)$ & $2.00(1.71)$ \\
\hline $2-3$ & $6686(0.35)$ & $2.05(1.75)$ \\
\hline $3-4$ & $4659(0.24)$ & $2.05(1.72)$ \\
\hline $4-5$ & $5151(0.27)$ & $1.98(1.70)$ \\
\hline $5-6$ & $9681(0.51)$ & $1.82(1.57)$ \\
\hline $6-7$ & $22,818(1.20)$ & $1.70(1.47)$ \\
\hline $7-8$ & $51,225(2.69)$ & $1.67(1.43)$ \\
\hline $8-9$ & $90,270(4.74)$ & $1.71(1.47)$ \\
\hline
\end{tabular}




\begin{tabular}{cll}
\hline Timeframe & Ratings, $\mathrm{n}(\%)$ & Mean rating, mean (SD) \\
\hline $9-10$ & $122,461(6.42)$ & $1.74(1.50)$ \\
$10-11$ & $144,834(7.60)$ & $1.75(1.51)$ \\
$11-12$ & $152,606(8.01)$ & $1.77(1.53)$ \\
$12-13$ & $143,618(7.53)$ & $1.78(1.54)$ \\
$13-14$ & $136,245(7.15)$ & $1.76(1.53)$ \\
$14-15$ & $129,596(6.80)$ & $1.74(1.50)$ \\
$15-16$ & $121,427(6.37)$ & $1.75(1.52)$ \\
$16-17$ & $116,451(6.11)$ & $1.76(1.53)$ \\
$17-18$ & $111,075(5.83)$ & $1.77(1.54)$ \\
$18-19$ & $101,968(5.35)$ & $1.75(1.53)$ \\
$19-20$ & $98,494(5.17)$ & $1.73(1.52)$ \\
$20-21$ & $95,222(5.00)$ & $1.72(1.51)$ \\
$21-22$ & $89,447(4.69)$ & $1.73(1.51)$ \\
$22-23$ & $71,515(3.75)$ & $1.78(1.54)$ \\
$23-24$ & $45,156(2.37)$ & $1.85(1.60)$ \\
\hline
\end{tabular}

\section{Number of Rated Physicians and Ratings Per Rated Physician}

Between 2010 and 2019, a total of 127,921 physicians were rated on jameda (see Table 2). The number of rated physicians increased constantly from 19,305 in 2010 to 82,511 in 2018. In 2019 , the number of rated physicians decreased to 73,071 rated physicians. The number of ratings that rated physicians received demonstrated an increasing trend. In 2010, 66.94\% $(12,923 / 19,305)$ of all rated physicians were rated only once, $30.88 \%(5961 / 19,305)$ were rated $2-5$ times, $1.71 \%(330 / 19,305)$ were rated 6-10 times, and $0.47 \%(91 / 19,305)$ were rated $11-50$ times. In $2019,40.84 \%(29,843 / 73,071)$ of all rated physicians were rated only once, $46.89 \%(34,262 / 73,071)$ were rated $2-5$ times, $8.21 \%(5998 / 73,071)$ were rated 6-10 times, $3.93 \%$ $(2875 / 73,071)$ were rated $11-50$ times, and $0.13 \%(93 / 73,071)$ were rated more than 50 times. Over the entire decade, $11.43 \%$ $(14,625 / 127,921)$ of all rated physicians were rated once, and $4.23 \%(5413 / 127,921)$ were rated more than 50 times. Please note that the overall numbers cannot be summed up here. For example, one physician received 1 rating in 2010, 3 ratings in 2011, 5 ratings in 2013, 1 rating in 2015, 11 ratings in 2015, 23 ratings in 2017, and 19 ratings in 2019. In sum, this physician was rated 63 times and would be assigned to the category " $\geq 51$ Ratings." Similarly, the overall average number of ratings per rated physician increased from 1.65 (SD 1.56) in 2010 to 3.19 (SD 4.69) in 2019. Comparing the number of ratings and rated physicians with the total number of physicians in the German outpatient sector [43], in 2010, 13.64\% $(19,305 / 141,461)$ of all physicians had been rated on jameda, $21.93 \%(31,335 / 142,855)$ in $2011,29.22 \%(42,089 / 144,058)$ in $2012,36.36 \%$ $(53,065 / 145,933)$ in $2013,42.71 \%(63,182 / 147,948)$ in 2014 , $45.56 \%(68,392 / 150,106)$ in $2015,50.51 \%(76,773 / 151,989)$ in $2016,51.69 \%(79,799 / 154,369)$ in 2017 , and $52.46 \%$ $(82,511 / 157,288)$ in 2018 (see also Multimedia Appendix 1). Thus, more than half of all German physicians have been rated online on jameda each year in Germany since 2016. 
Table 2. Overall ratings on jameda between 2010 and 2019.

\begin{tabular}{|c|c|c|c|c|c|c|c|c|c|c|c|}
\hline \multirow[t]{2}{*}{ Ratings } & \multicolumn{10}{|l|}{ Year } & \multirow[t]{2}{*}{$\begin{array}{l}\text { Overall } \\
(\mathrm{n}=127,921)\end{array}$} \\
\hline & $\begin{array}{l}2010 \\
(\mathrm{n}=19,305)\end{array}$ & $\begin{array}{l}2011 \\
(n=31,336)\end{array}$ & $\begin{array}{l}2012 \\
(n=42,089)\end{array}$ & $\begin{array}{l}2013 \\
(\mathrm{n}=53,065)\end{array}$ & $\begin{array}{l}2014 \\
(\mathrm{n}=63,182)\end{array}$ & $\begin{array}{l}2015 \\
(\mathrm{n}=68,392)\end{array}$ & $\begin{array}{l}2016 \\
(n=76,773)\end{array}$ & $\begin{array}{l}2017 \\
(\mathrm{n}=79,799)\end{array}$ & $\begin{array}{l}2018 \\
(\mathrm{n}=82,511)\end{array}$ & $\begin{array}{l}2019 \\
(\mathrm{n}=73,071)\end{array}$ & \\
\hline \multicolumn{12}{|c|}{ Overall number and percentage of rated physicians, $n(\%)$} \\
\hline 1 rating & $\begin{array}{l}12,923 \\
(66.94)\end{array}$ & $\begin{array}{l}18,256 \\
(58.26)\end{array}$ & $\begin{array}{l}21,133 \\
(50.21)\end{array}$ & $\begin{array}{l}22,177 \\
(41.79)\end{array}$ & $\begin{array}{l}22,229 \\
(35.18)\end{array}$ & $\begin{array}{l}24,512 \\
(35.84)\end{array}$ & $\begin{array}{l}25,859 \\
(33.68)\end{array}$ & $\begin{array}{l}26,810 \\
(33.60)\end{array}$ & $\begin{array}{l}28,971 \\
(35.11)\end{array}$ & $\begin{array}{l}29,843 \\
(40.84)\end{array}$ & $\begin{array}{l}14,625 \\
(11.43)\end{array}$ \\
\hline $2-5$ ratings & $\begin{array}{l}5961 \\
(30.88)\end{array}$ & $\begin{array}{l}11,877 \\
(37.90)\end{array}$ & $\begin{array}{l}18,389 \\
(43.69)\end{array}$ & $\begin{array}{l}25,321 \\
(47.71)\end{array}$ & $\begin{array}{l}31,422 \\
(49.73)\end{array}$ & $\begin{array}{l}33,751 \\
(49.35)\end{array}$ & $\begin{array}{l}38,263 \\
(49.84)\end{array}$ & $\begin{array}{l}39,808 \\
(49.89)\end{array}$ & $\begin{array}{l}40,602 \\
(49.21)\end{array}$ & $\begin{array}{l}34,262 \\
(46.89)\end{array}$ & $\begin{array}{l}31,507 \\
(24.63)\end{array}$ \\
\hline $\begin{array}{l}\text { 6-10 rat- } \\
\text { ings }\end{array}$ & $\begin{array}{l}330 \\
(1.71)\end{array}$ & $\begin{array}{l}933 \\
(2.98)\end{array}$ & $\begin{array}{l}1936 \\
(4.60)\end{array}$ & $\begin{array}{l}4085 \\
(7.70)\end{array}$ & $\begin{array}{l}6755 \\
(10.69)\end{array}$ & $\begin{array}{l}7061 \\
(10.32)\end{array}$ & $\begin{array}{l}8710 \\
(11.35)\end{array}$ & $\begin{array}{l}9099 \\
(11.40)\end{array}$ & $\begin{array}{l}9007 \\
(10.92)\end{array}$ & $\begin{array}{l}5998 \\
(8.21)\end{array}$ & $\begin{array}{l}26,285 \\
(20.55)\end{array}$ \\
\hline $\begin{array}{l}11-50 \text { rat- } \\
\text { ings }\end{array}$ & $91(0.47)$ & $\begin{array}{l}259 \\
(0.83)\end{array}$ & $\begin{array}{l}604 \\
(1.44)\end{array}$ & $\begin{array}{l}1424 \\
(2.68)\end{array}$ & $\begin{array}{l}2683 \\
(4.25)\end{array}$ & $\begin{array}{l}2954 \\
(4.32)\end{array}$ & $\begin{array}{l}3787 \\
(4.93)\end{array}$ & $\begin{array}{l}3933 \\
(4.93)\end{array}$ & $\begin{array}{l}3801 \\
(4.61)\end{array}$ & $\begin{array}{l}2875 \\
(3.93)\end{array}$ & $\begin{array}{l}50,091 \\
(39.16)\end{array}$ \\
\hline$\geq 51$ ratings & $0(0.00)$ & $11(0.00)$ & $27(0.01)$ & $58(0.11)$ & $93(0.15)$ & $\begin{array}{l}114 \\
(0.12)\end{array}$ & $\begin{array}{l}154 \\
(0.20)\end{array}$ & $\begin{array}{l}149 \\
(0.19)\end{array}$ & $\begin{array}{l}130 \\
(0.16)\end{array}$ & $93(0.13)$ & $\begin{array}{l}5413 \\
(4.23)\end{array}$ \\
\hline $\begin{array}{l}\text { Percentage of } \\
\text { rated physi- } \\
\text { cians, } \%(\mathrm{~N})\end{array}$ & $\begin{array}{l}13.64 \\
(141,461)\end{array}$ & $\begin{array}{l}21.93 \\
(142,855)\end{array}$ & $\begin{array}{l}29.22 \\
(144,058)\end{array}$ & $\begin{array}{l}36.36 \\
(145,933)\end{array}$ & $\begin{array}{l}42.71 \\
(147,948)\end{array}$ & $\begin{array}{l}45.56 \\
(150,106)\end{array}$ & $\begin{array}{l}50.51 \\
(151,989)\end{array}$ & $\begin{array}{l}51.69 \\
(154,369)\end{array}$ & $\begin{array}{l}52.46 \\
(157,288)\end{array}$ & N/A ${ }^{a}$ & $-{ }^{b}$ \\
\hline \multicolumn{12}{|c|}{ Number of ratings per rated physician } \\
\hline Mean (SD) & $\begin{array}{l}1.65 \\
(1.56)\end{array}$ & $\begin{array}{l}1.97 \\
(2.51)\end{array}$ & $\begin{array}{l}2.33 \\
(3.22)\end{array}$ & $\begin{array}{l}2.90 \\
(4.05)\end{array}$ & $\begin{array}{l}3.47 \\
(4.84)\end{array}$ & $\begin{array}{l}3.47 \\
(4.95)\end{array}$ & $\begin{array}{l}3.71 \\
(5.43)\end{array}$ & $\begin{array}{l}3.68 \\
(5.09)\end{array}$ & $\begin{array}{l}3.55 \\
(4.92)\end{array}$ & $\begin{array}{l}3.19 \\
(4.69)\end{array}$ & $\begin{array}{l}14.90 \\
(24.04)\end{array}$ \\
\hline Maximum & 39 & 137 & 151 & 149 & 165 & 154 & 197 & 143 & 215 & 148 & 943 \\
\hline
\end{tabular}

${ }^{\mathrm{a}} \mathrm{N} / \mathrm{A}$ : not available.

${ }^{\mathrm{b}}$ Not applicable.

\section{Rating Evaluations}

Of the 1,906,146 ratings posted between 2010 and $2019,75.16 \%$ $(1,432,624 / 1,906,146)$ of all ratings were in the best rating category of "very good," and 5.66\% $(107,912 / 1,906,146)$ of the ratings were in the lowest category of "insufficient" (see Table 3 ). Furthermore, the percentage of ratings on both ends of the rating scale increased over time, from $71.95 \%$ (2010) to $78.17 \%$ (2019) for very positive ratings and from $3.91 \%$ (2010) to $6.12 \%$ (2019) for very negative ratings. However, the overall average rating remained relatively constant. The average rating was 1.73 (SD 1.42) in 2010 and 1.71 (SD 1.52) in 2019, with an overall average of 1.76 (SD 1.53).
With regards to the correlation between the average rating of a rated physician and the number of ratings per physician, physicians with a lower number of ratings tended to receive ratings across the rating scale, while physicians with a higher number of ratings tended to have better ratings (see Figure 1). Physicians with a single rating had a mean rating of 1.58 (SD 1.28). Afterwards, mean ratings get worse with increasing number of ratings. Physicians with between 21 and 50 online ratings received the worst ratings (mean 1.95, SD 0.84). Mean ratings then improve, with physicians having 51-100 ratings receiving a mean rating of 1.79 (SD 0.86) and physicians with more than 100 ratings receiving the best ratings (mean 1.34, SD 0.47; see Table 4). 
Table 3. Overall rating evaluations on jameda between 2010 and 2019.

\begin{tabular}{|c|c|c|c|c|c|c|c|c|c|c|c|}
\hline \multirow{2}{*}{$\begin{array}{l}\text { Overall rating } \\
\text { evaluation }\end{array}$} & \multicolumn{10}{|l|}{ Year } & \multirow{2}{*}{$\begin{array}{l}\text { Overall } \\
(\mathrm{n}=1,906,146)\end{array}$} \\
\hline & $\begin{array}{l}2010 \\
(n=31,908)\end{array}$ & $\begin{array}{l}2011 \\
(n=61,726)\end{array}$ & $\begin{array}{l}2012 \\
(n=98,041)\end{array}$ & $\begin{array}{l}2013 \\
(\mathrm{n}=154,119)\end{array}$ & $\begin{array}{l}2014 \\
(\mathrm{n}=219,319)\end{array}$ & $\begin{array}{l}2015 \\
(n=237,354)\end{array}$ & $\begin{array}{l}2016 \\
(n=284,475)\end{array}$ & $\begin{array}{l}2017 \\
(\mathrm{n}=293,744)\end{array}$ & $\begin{array}{l}2018 \\
(\mathrm{n}=292,721)\end{array}$ & $\begin{array}{l}2019 \\
(\mathrm{n}=232,729)\end{array}$ & \\
\hline \multicolumn{12}{|c|}{ Rating based on the 6-point grading system, n (\%) } \\
\hline $\begin{array}{l}\text { 1=very } \\
\text { good }\end{array}$ & $\begin{array}{l}22,957 \\
(71.95)\end{array}$ & $\begin{array}{l}44,952 \\
(72.83)\end{array}$ & $\begin{array}{l}72,066 \\
(73.51)\end{array}$ & $\begin{array}{l}111,043 \\
(72.05)\end{array}$ & $\begin{array}{l}160,263 \\
(73.07)\end{array}$ & $\begin{array}{l}175,416 \\
(73.90)\end{array}$ & $\begin{array}{l}217,533 \\
(76.47)\end{array}$ & $\begin{array}{l}224,527 \\
(76.44)\end{array}$ & $\begin{array}{l}221,951 \\
(75.82)\end{array}$ & $\begin{array}{l}181,916 \\
(78.17)\end{array}$ & $\begin{array}{l}1,432,624 \\
(75.16)\end{array}$ \\
\hline $2=\operatorname{good}$ & $\begin{array}{l}3406 \\
(10.67)\end{array}$ & $\begin{array}{l}5783 \\
(9.37)\end{array}$ & $\begin{array}{l}7889 \\
(8.05)\end{array}$ & $\begin{array}{l}12,113 \\
(7.86)\end{array}$ & $\begin{array}{l}16,651 \\
(7.59)\end{array}$ & $\begin{array}{l}17,328 \\
(7.30)\end{array}$ & $\begin{array}{l}19,383 \\
(6.81)\end{array}$ & $\begin{array}{l}18,489 \\
(6.29)\end{array}$ & $\begin{array}{l}17,205 \\
(5.88)\end{array}$ & $\begin{array}{l}12,203 \\
(5.24)\end{array}$ & $\begin{array}{l}130,450 \\
(6.84)\end{array}$ \\
\hline $\begin{array}{l}3=\text { satisfac- } \\
\text { tory }\end{array}$ & $\begin{array}{l}1036 \\
(3.25)\end{array}$ & $\begin{array}{l}2007 \\
(3.25)\end{array}$ & $\begin{array}{l}2920 \\
(2.98)\end{array}$ & $\begin{array}{l}4766 \\
(3.09)\end{array}$ & $\begin{array}{l}6200 \\
(2.83)\end{array}$ & $\begin{array}{l}6321 \\
(2.66)\end{array}$ & $\begin{array}{l}6655 \\
(2.34)\end{array}$ & $\begin{array}{l}6848 \\
(2.33)\end{array}$ & $\begin{array}{l}6665 \\
(2.28)\end{array}$ & $\begin{array}{l}4491 \\
(1.93)\end{array}$ & $\begin{array}{l}47,909 \\
(2.51)\end{array}$ \\
\hline $4=$ fair & $\begin{array}{l}1312 \\
(4.11)\end{array}$ & $\begin{array}{l}2635 \\
(4.27)\end{array}$ & $\begin{array}{l}4082 \\
(4.16)\end{array}$ & $\begin{array}{l}6631 \\
(4.30)\end{array}$ & $\begin{array}{l}9073 \\
(4.14)\end{array}$ & $\begin{array}{l}9444 \\
(3.98)\end{array}$ & $\begin{array}{l}9545 \\
(3.36)\end{array}$ & $\begin{array}{l}9678 \\
(3.29)\end{array}$ & $\begin{array}{l}10,021 \\
(3.42)\end{array}$ & $\begin{array}{l}7056 \\
(3.03)\end{array}$ & $\begin{array}{l}69,477 \\
(3.64)\end{array}$ \\
\hline $5=$ deficient & $\begin{array}{l}1948 \\
(6.11)\end{array}$ & $\begin{array}{l}3910 \\
(6.33)\end{array}$ & $\begin{array}{l}6233 \\
(6.36)\end{array}$ & $\begin{array}{l}10,694 \\
(6.94)\end{array}$ & $\begin{array}{l}15,121 \\
(6.89)\end{array}$ & $\begin{array}{l}15,658 \\
(6.60)\end{array}$ & $\begin{array}{l}16,493 \\
(5.80)\end{array}$ & $\begin{array}{l}17,339 \\
(5.90)\end{array}$ & $\begin{array}{l}17,537 \\
(5.99)\end{array}$ & $\begin{array}{l}12,841 \\
(5.52)\end{array}$ & $\begin{array}{l}117,774 \\
(6.18)\end{array}$ \\
\hline $\begin{array}{l}6=\text { insuffi- } \\
\text { cient }\end{array}$ & $\begin{array}{l}1249 \\
(3.91)\end{array}$ & $\begin{array}{l}2439 \\
(3.95)\end{array}$ & $\begin{array}{l}4851 \\
(4.95)\end{array}$ & $\begin{array}{l}8872 \\
(5.76)\end{array}$ & $\begin{array}{l}12,011 \\
(5.48)\end{array}$ & $\begin{array}{l}13,187 \\
(5.56)\end{array}$ & $\begin{array}{l}14,866 \\
(5.23)\end{array}$ & $\begin{array}{l}16,863 \\
(5.74)\end{array}$ & $\begin{array}{l}19,342 \\
(6.61)\end{array}$ & $\begin{array}{l}14,232 \\
(6.12)\end{array}$ & $\begin{array}{l}107,912 \\
(5.66)\end{array}$ \\
\hline Mean (SD) & $\begin{array}{l}1.73 \\
(1.42)\end{array}$ & $\begin{array}{l}1.74 \\
(1.44)\end{array}$ & $\begin{array}{l}1.77 \\
(1.50)\end{array}$ & $\begin{array}{l}1.83 \\
(1.56)\end{array}$ & $\begin{array}{l}1.81 \\
(1.54)\end{array}$ & $\begin{array}{l}1.79 \\
(1.54)\end{array}$ & $\begin{array}{l}1.71 \\
(1.48)\end{array}$ & $\begin{array}{l}1.73 \\
(1.52)\end{array}$ & $\begin{array}{l}1.78 \\
(1.57)\end{array}$ & $\begin{array}{l}1.71 \\
(1.52)\end{array}$ & $\begin{array}{l}1.76 \\
(1.53)\end{array}$ \\
\hline
\end{tabular}

Figure 1. Scatterplot (bivariate) of the number of ratings per physician with the mean overall performance for rated physicians.

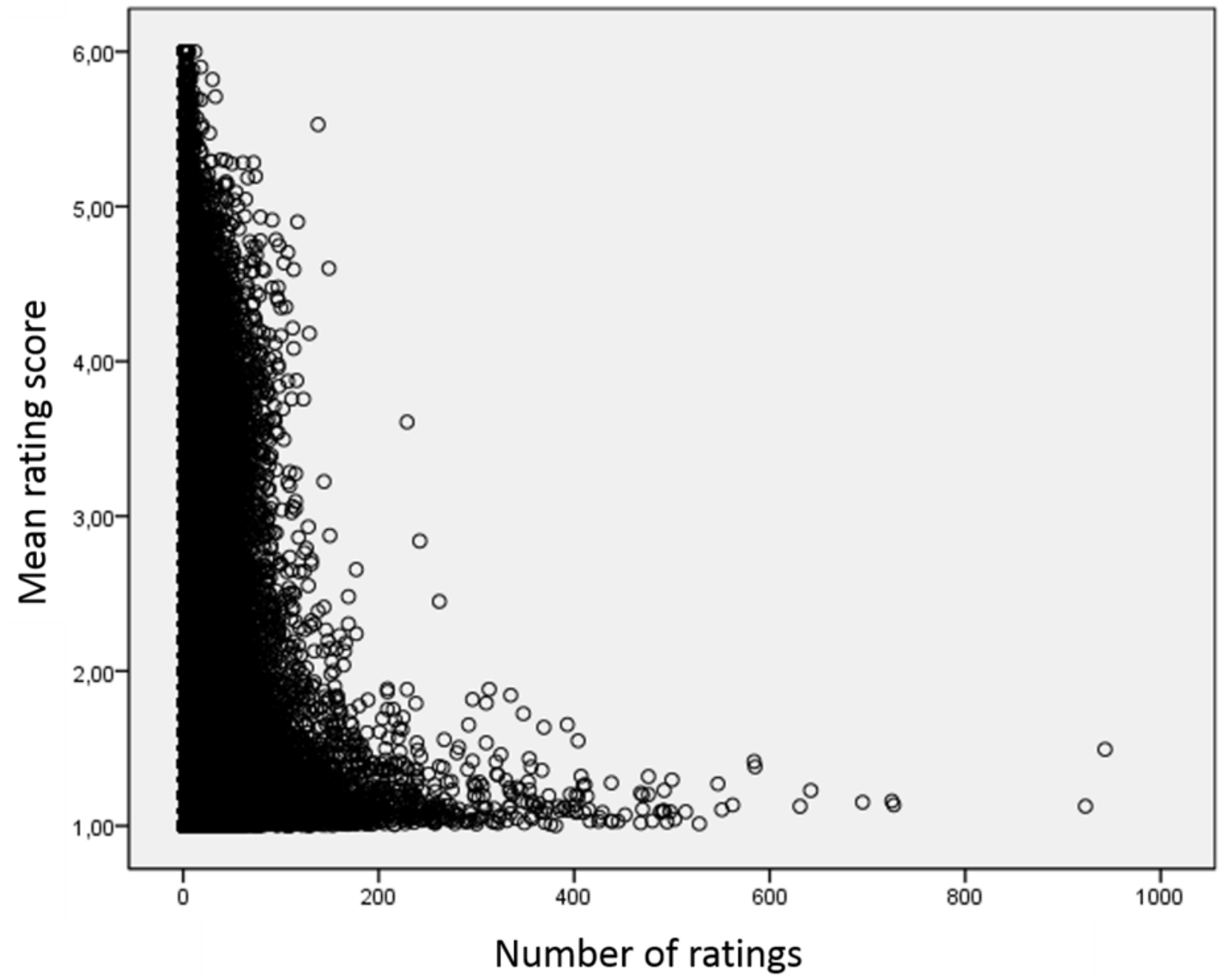


Table 4. Online rating results by the number of ratings per physician.

\begin{tabular}{|c|c|c|c|c|c|c|c|}
\hline \multirow{2}{*}{$\begin{array}{l}\text { Number of ratings per } \\
\text { physician }\end{array}$} & \multirow{2}{*}{$\begin{array}{l}\text { Average rating, } \\
\text { mean (SD) }\end{array}$} & \multicolumn{6}{|c|}{ Rating based on the 6-point grading system ${ }^{\mathrm{a}}, \mathrm{n}(\%)$} \\
\hline & & 1 & 2 & 3 & 4 & 5 & 6 \\
\hline $1(\mathrm{n}=14,625)$ & $1.58(1.28)$ & $11,741(80.28)$ & $1023(6.99)$ & $302(2.06)$ & $394(2.69)$ & $645(4.41)$ & $520(3.56)$ \\
\hline $2-5(n=31,505)$ & $1.67(0.96)$ & $19,733(62.63)$ & $6204(19.69)$ & $3477(11.04)$ & $1395(4.43)$ & $471(1.50)$ & $225(0.71)$ \\
\hline $6-10(n=26,258)$ & $1.76(0.81)$ & $12,505(47.62)$ & $9459(36.02)$ & $3096(11.79)$ & $961(3.66)$ & $214(0.81)$ & $23(0.09)$ \\
\hline $11-20(n=29,049)$ & $1.86(0.78)$ & $12,243(42.15)$ & $11,188(38.51)$ & $4289(14.76)$ & $1162(4.00)$ & $159(0.55)$ & $8(0.02)$ \\
\hline $21-50(n=20,658)$ & $1.95(0.84)$ & $8044(38.94)$ & $7833(37.92)$ & $3408(16.50)$ & 1195 (5.78) & $176(0.85)$ & $2(0.00)$ \\
\hline $51-100(\mathrm{n}=3933)$ & $1.79(0.86)$ & 2084 (52.99) & $1122(28.53)$ & 446 (11.34) & $246(6.25)$ & $35(0.89)$ & $0(0.00)$ \\
\hline$>100(n=1445)$ & $1.34(0.47)$ & $1181(81.73)$ & $220(15.22)$ & 28 (1.94) & $10(0.69)$ & $5(0.35)$ & $1(0.07)$ \\
\hline Total $(\mathrm{n}=127,473)$ & $1.77(0.92)$ & $67,531(52.98)$ & $37,049(29.06)$ & $15,046(11.80)$ & $5363(4.21)$ & 1705 (1.34) & $779(0.61)$ \\
\hline
\end{tabular}

${ }^{\mathrm{a}} 1=$ very good, $2=$ good, $3=$ satisfactory, $4=$ =fair, $5=$ deficient, and $6=$ insufficient.

\section{Ratings by Medical Specialty}

Between 2010 and 2019, general practitioners $(343,242)$, internists $(266,899)$, gynecologists $(232,914)$, and orthopedists $(229,481)$ received the highest number of ratings, while pediatricians $(87,330)$, ophthalmologists $(79,699)$, and urologists $(63,703)$ received the lowest number of ratings (see Table 5). However, according to the relative distribution of ratings, the most frequently rated medical specialties in 2018 were orthopedists $(6160 / 7302,84.36 \%)$; oral maxillofacial surgeons (1017/1257, 80.91\%); ear, nose, and throat (ENT) specialists
(3559/4479, 79.46\%); and dermatologists (3562/4632, 76.90\%). In contrast, the least frequently rated medical specialties were radiologists $(863 / 4078,21.16 \%)$ and anesthesiologists (601/4247, 14.15\%; see Multimedia Appendix 2). Among the 10 most frequently rated medical specialties, the best rated medical specialties were urologists (mean 1.50, SD 1.29), general practitioners (mean 1.64, SD 1.40), and internists (mean $1.68, \mathrm{SD} 1.45)$. The lowest ratings were given to pediatricians (mean 1.92, SD 1.62), ophthalmologists (mean 2.06, SD 1.74), and dermatologists (mean 2.11, SD 1.77). 
Table 5. Ratings by medical specialty.

\begin{tabular}{|c|c|c|c|c|c|c|c|c|c|c|c|}
\hline \multirow{2}{*}{$\begin{array}{l}\text { Medical } \\
\text { specialty }\end{array}$} & \multicolumn{10}{|l|}{ Year } & \multirow{2}{*}{$\begin{array}{l}\text { Overall } \\
(\mathrm{n}=127,921 \\
\text { rated } \\
\text { physi- } \\
\text { cians; } \\
\mathrm{n}=1,906,146 \\
\text { ratings) }\end{array}$} \\
\hline & $\begin{array}{l}2010 \\
(\mathrm{n}=19,305 \\
\text { rated } \\
\text { physi- } \\
\text { cians; } \\
\mathrm{n}=31,908 \\
\text { ratings) }\end{array}$ & $\begin{array}{l}2011 \\
(\mathrm{n}=31,336 \\
\text { rated } \\
\text { physi- } \\
\text { cians; } \\
\mathrm{n}=61,726 \\
\text { ratings) }\end{array}$ & $\begin{array}{l}2012 \\
(\mathrm{n}=42,089 \\
\text { rated } \\
\text { physi- } \\
\text { cians; } \\
\mathrm{n}=98,041 \\
\text { ratings) }\end{array}$ & $\begin{array}{l}2013 \\
(\mathrm{n}=53,065 \\
\text { rated } \\
\text { physi- } \\
\text { cians; } \\
\mathrm{n}=154,119 \\
\text { ratings) }\end{array}$ & $\begin{array}{l}2014 \\
(\mathrm{n}=63,182 \\
\text { rated } \\
\text { physi- } \\
\text { cians; } \\
\mathrm{n}=219,319 \\
\text { ratings) }\end{array}$ & $\begin{array}{l}2015 \\
(\mathrm{n}=68,392 \\
\text { rated } \\
\text { physi- } \\
\text { cians; } \\
\mathrm{n}=237,354 \\
\text { ratings) }\end{array}$ & $\begin{array}{l}2016 \\
(\mathrm{n}=76,773 \\
\text { rated } \\
\text { physi- } \\
\text { cians; } \\
\mathrm{n}=284,475 \\
\text { ratings) }\end{array}$ & $\begin{array}{l}2017 \\
(\mathrm{n}=79,799 \\
\text { rated } \\
\text { physi- } \\
\text { cians; } \\
\mathrm{n}=293,744 \\
\text { ratings) }\end{array}$ & $\begin{array}{l}2018 \\
(\mathrm{n}=82,551 \\
\text { rated } \\
\text { physi- } \\
\text { cians; } \\
\mathrm{n}=292,721 \\
\text { ratings) }\end{array}$ & $\begin{array}{l}2019 \\
(\mathrm{n}=73,071 \\
\text { rated } \\
\text { physi- } \\
\text { cians; } \\
\mathrm{n}=232,729 \\
\text { ratings) }\end{array}$ & \\
\hline \multicolumn{12}{|c|}{ General practitioner } \\
\hline $\begin{array}{l}\text { Rated } \\
\text { physicians, } \\
\text { n }(\%)\end{array}$ & $\begin{array}{l}4891 \\
(25.34)\end{array}$ & $\begin{array}{l}8161 \\
(26.04)\end{array}$ & $\begin{array}{l}10,533 \\
(25.03)\end{array}$ & $\begin{array}{l}13,077 \\
(24.64)\end{array}$ & $\begin{array}{l}15,767 \\
(24.95)\end{array}$ & $\begin{array}{l}17,016 \\
(24.88)\end{array}$ & $\begin{array}{l}19,289 \\
(25.12)\end{array}$ & $\begin{array}{l}19,586 \\
(24.54)\end{array}$ & $\begin{array}{l}19,967 \\
(24.19)\end{array}$ & $\begin{array}{l}16,818 \\
(23.02)\end{array}$ & $\begin{array}{l}33,414 \\
(26.12)\end{array}$ \\
\hline $\begin{array}{l}\text { Number of } \\
\text { ratings }\end{array}$ & $\begin{array}{l}7241 \\
(22.69)\end{array}$ & $\begin{array}{l}13,737 \\
(22.25)\end{array}$ & $\begin{array}{l}19,210 \\
(19.59)\end{array}$ & $\begin{array}{l}27,952 \\
(18.14)\end{array}$ & $\begin{array}{l}39,914 \\
(18.20)\end{array}$ & $\begin{array}{l}42,188 \\
(17.77)\end{array}$ & $\begin{array}{l}51,504 \\
(18.10)\end{array}$ & $\begin{array}{l}51,725 \\
(17.61)\end{array}$ & $\begin{array}{l}51,682 \\
(17.66)\end{array}$ & $\begin{array}{l}38,089 \\
(16.37)\end{array}$ & $\begin{array}{l}343,242 \\
(18.01)\end{array}$ \\
\hline $\begin{array}{l}\text { Mean rat- } \\
\operatorname{ing}(\mathrm{SD})^{\mathrm{a}} \\
\mathrm{n}(\%)\end{array}$ & $\begin{array}{l}1.55 \\
(1.21)\end{array}$ & $\begin{array}{l}1.53 \\
(1.21)\end{array}$ & $\begin{array}{l}1.54 \\
(1.24)\end{array}$ & $\begin{array}{l}1.60 \\
(1.33)\end{array}$ & $\begin{array}{l}1.61 \\
(1.35)\end{array}$ & $\begin{array}{l}1.66 \\
(1.40)\end{array}$ & $\begin{array}{l}1.59 \\
(1.35)\end{array}$ & $\begin{array}{l}1.65 \\
(1.43)\end{array}$ & $\begin{array}{l}1.73 \\
(1.52)\end{array}$ & $\begin{array}{l}1.71 \\
(1.51)\end{array}$ & $\begin{array}{l}1.64 \\
(1.40)\end{array}$ \\
\hline \multicolumn{12}{|l|}{ Internist } \\
\hline $\begin{array}{l}\text { Rated } \\
\text { physicians, } \\
\text { n }(\%)\end{array}$ & $\begin{array}{l}3230 \\
(16.73)\end{array}$ & $\begin{array}{l}5286 \\
(16.87)\end{array}$ & $\begin{array}{l}6897 \\
(16.39)\end{array}$ & $\begin{array}{l}8779 \\
(16.54)\end{array}$ & $\begin{array}{l}10,635 \\
(16.83)\end{array}$ & $\begin{array}{l}11,511 \\
(16.83)\end{array}$ & $\begin{array}{l}13,374 \\
(17.42)\end{array}$ & $\begin{array}{l}13,849 \\
(17.35)\end{array}$ & $\begin{array}{l}14,634 \\
(17.73)\end{array}$ & $\begin{array}{l}13,306 \\
(18.21)\end{array}$ & $\begin{array}{l}23,734 \\
(18.55)\end{array}$ \\
\hline $\begin{array}{l}\text { Mean rat- } \\
\text { ing }(\mathrm{SD})^{\mathrm{a}}\end{array}$ & $\begin{array}{l}1.59 \\
(1.27)\end{array}$ & $\begin{array}{l}1.62 \\
(1.32)\end{array}$ & $\begin{array}{l}1.62 \\
(1.36)\end{array}$ & $\begin{array}{l}1.70 \\
(1.44)\end{array}$ & $\begin{array}{l}1.68 \\
(1.43)\end{array}$ & $\begin{array}{l}1.70 \\
(1.46)\end{array}$ & $\begin{array}{l}1.63 \\
(1.40)\end{array}$ & $\begin{array}{l}1.68 \\
(1.47)\end{array}$ & $\begin{array}{l}1.73 \\
(1.53)\end{array}$ & $\begin{array}{l}1.68 \\
(1.49)\end{array}$ & $\begin{array}{l}1.68 \\
(1.45)\end{array}$ \\
\hline \multicolumn{12}{|l|}{ Gynecologist } \\
\hline $\begin{array}{l}\text { Rated } \\
\text { physicians, } \\
\text { n }(\%)\end{array}$ & $\begin{array}{l}2157 \\
(11.17)\end{array}$ & $\begin{array}{l}3568 \\
(11.39)\end{array}$ & $\begin{array}{l}5084 \\
(12.08)\end{array}$ & $\begin{array}{l}6291 \\
(11.86)\end{array}$ & $\begin{array}{l}7163 \\
(11.34)\end{array}$ & $\begin{array}{l}7602 \\
(11.12)\end{array}$ & $\begin{array}{l}8165 \\
(10.64)\end{array}$ & $\begin{array}{l}8445 \\
(10.58)\end{array}$ & $\begin{array}{l}8653 \\
(10.48)\end{array}$ & $\begin{array}{l}7650 \\
(10.47)\end{array}$ & $\begin{array}{l}11,598 \\
(9.07)\end{array}$ \\
\hline $\begin{array}{l}\text { Number of } \\
\text { ratings, } \mathrm{n} \\
(\%)\end{array}$ & $\begin{array}{l}3901 \\
(12.23)\end{array}$ & $\begin{array}{l}7800 \\
(12.64)\end{array}$ & $\begin{array}{l}13,987 \\
(14.27)\end{array}$ & $\begin{array}{l}21,880 \\
(14.20)\end{array}$ & $\begin{array}{l}28,672 \\
(13.07)\end{array}$ & $\begin{array}{l}29,795 \\
(12.55)\end{array}$ & $\begin{array}{l}33,862 \\
(11.90)\end{array}$ & $\begin{array}{l}34,530 \\
(11.76)\end{array}$ & $\begin{array}{l}33,562 \\
(11.47)\end{array}$ & $\begin{array}{l}24,925 \\
(10.71)\end{array}$ & $\begin{array}{l}232,914 \\
(12.22)\end{array}$ \\
\hline $\begin{array}{l}\text { Mean rat- } \\
\text { ing }(\mathrm{SD})^{\mathrm{a}}\end{array}$ & $\begin{array}{l}1.66 \\
(1.36)\end{array}$ & $\begin{array}{l}1.64 \\
(1.33)\end{array}$ & $\begin{array}{l}1.69 \\
(1.41)\end{array}$ & $\begin{array}{l}1.79 \\
(1.49)\end{array}$ & $\begin{array}{l}1.79 \\
(1.50)\end{array}$ & $\begin{array}{l}1.80 \\
(1.51)\end{array}$ & $\begin{array}{l}1.74 \\
(1.49)\end{array}$ & $\begin{array}{l}1.73 \\
(1.48)\end{array}$ & $\begin{array}{l}1.76 \\
(1.52)\end{array}$ & $\begin{array}{l}1.69 \\
(1.47)\end{array}$ & $\begin{array}{l}1.75 \\
(1.48)\end{array}$ \\
\hline \multicolumn{12}{|l|}{ Orthopedist } \\
\hline $\begin{array}{l}\text { Rated } \\
\text { physicians, } \\
\text { n }(\%)\end{array}$ & $\begin{array}{l}1662 \\
(8.61)\end{array}$ & $\begin{array}{l}2548 \\
(8.13)\end{array}$ & $\begin{array}{l}3333 \\
(7.92)\end{array}$ & $\begin{array}{l}4007 \\
(7.55)\end{array}$ & $\begin{array}{l}4629 \\
(7.33)\end{array}$ & $\begin{array}{l}5051 \\
(7.39)\end{array}$ & $\begin{array}{l}5579 \\
(7.27)\end{array}$ & $\begin{array}{l}5907 \\
(7.40)\end{array}$ & $\begin{array}{l}6160 \\
(7.46)\end{array}$ & $\begin{array}{l}5894 \\
(8.07)\end{array}$ & $\begin{array}{l}8022 \\
(6.27)\end{array}$ \\
\hline $\begin{array}{l}\text { Number of } \\
\text { ratings, } \mathrm{n} \\
(\%)\end{array}$ & $\begin{array}{l}3412 \\
(10.69)\end{array}$ & $\begin{array}{l}6836 \\
(11.07)\end{array}$ & $\begin{array}{l}11,020 \\
(11.24)\end{array}$ & $\begin{array}{l}17,805 \\
(11.55)\end{array}$ & $\begin{array}{l}25,714 \\
(11.72)\end{array}$ & $\begin{array}{l}28,876 \\
(12.17)\end{array}$ & $\begin{array}{l}34,242 \\
(12.04)\end{array}$ & $\begin{array}{l}36,416 \\
(12.40)\end{array}$ & $\begin{array}{l}35,564 \\
(12.15)\end{array}$ & $\begin{array}{l}29,596 \\
(12.72)\end{array}$ & $\begin{array}{l}229,481 \\
(12.04)\end{array}$ \\
\hline $\begin{array}{l}\text { Mean rat- } \\
\text { ing }(\mathrm{SD})^{\mathrm{a}}\end{array}$ & $\begin{array}{l}2.08 \\
(1.67)\end{array}$ & $\begin{array}{l}2.06 \\
(1.67)\end{array}$ & $\begin{array}{l}2.12 \\
(1.75)\end{array}$ & $\begin{array}{l}2.15 \\
(1.78)\end{array}$ & $\begin{array}{l}2.05 \\
(1.72)\end{array}$ & $\begin{array}{l}1.93 \\
(1.65)\end{array}$ & $\begin{array}{l}1.82 \\
(1.58)\end{array}$ & $\begin{array}{l}1.80 \\
(1.57)\end{array}$ & $\begin{array}{l}1.82 \\
(1.60)\end{array}$ & $\begin{array}{l}1.70 \\
(1.52)\end{array}$ & $\begin{array}{l}1.89 \\
(1.63)\end{array}$ \\
\hline \multicolumn{12}{|c|}{ Dermatologist (including venereologist) } \\
\hline $\begin{array}{l}\text { Rated } \\
\text { physicians, } \\
\text { n }(\%)\end{array}$ & $\begin{array}{l}855 \\
(4.43)\end{array}$ & $\begin{array}{l}1354 \\
(4.32)\end{array}$ & $\begin{array}{l}1947 \\
(4.63)\end{array}$ & $\begin{array}{l}2467 \\
(4.65)\end{array}$ & $\begin{array}{l}2811 \\
(4.45)\end{array}$ & $\begin{array}{l}3003 \\
(4.39)\end{array}$ & $\begin{array}{l}3229 \\
(4.21)\end{array}$ & $\begin{array}{l}3415 \\
(4.28)\end{array}$ & $\begin{array}{l}3562 \\
(4.31)\end{array}$ & $\begin{array}{l}3232 \\
(4.42)\end{array}$ & $\begin{array}{l}4517 \\
(3.53)\end{array}$ \\
\hline
\end{tabular}




\begin{tabular}{|c|c|c|c|c|c|c|c|c|c|c|c|}
\hline \multirow{2}{*}{$\begin{array}{l}\text { Medical } \\
\text { specialty }\end{array}$} & \multicolumn{10}{|l|}{ Year } & \multirow{2}{*}{$\begin{array}{l}\text { Overall } \\
(\mathrm{n}=127,921 \\
\text { rated } \\
\text { physi- } \\
\text { cians; } \\
\mathrm{n}=1,906,146 \\
\text { ratings })\end{array}$} \\
\hline & $\begin{array}{l}2010 \\
(\mathrm{n}=19,305 \\
\text { rated } \\
\text { physi- } \\
\text { cians; } \\
\mathrm{n}=31,908 \\
\text { ratings) }\end{array}$ & $\begin{array}{l}2011 \\
(\mathrm{n}=31,336 \\
\text { rated } \\
\text { physi- } \\
\text { cians; } \\
\mathrm{n}=61,726 \\
\text { ratings) }\end{array}$ & $\begin{array}{l}2012 \\
(\mathrm{n}=42,089 \\
\text { rated } \\
\text { physi- } \\
\text { cians; } \\
\mathrm{n}=98,041 \\
\text { ratings })\end{array}$ & $\begin{array}{l}2013 \\
(\mathrm{n}=53,065 \\
\text { rated } \\
\text { physi- } \\
\text { cians; } \\
\mathrm{n}=154,119 \\
\text { ratings })\end{array}$ & $\begin{array}{l}2014 \\
(\mathrm{n}=63,182 \\
\text { rated } \\
\text { physi- } \\
\text { cians; } \\
\mathrm{n}=219,319 \\
\text { ratings) }\end{array}$ & $\begin{array}{l}2015 \\
(\mathrm{n}=68,392 \\
\text { rated } \\
\text { physi- } \\
\text { cians; } \\
\mathrm{n}=237,354 \\
\text { ratings })\end{array}$ & $\begin{array}{l}2016 \\
(\mathrm{n}=76,773 \\
\text { rated } \\
\text { physi- } \\
\text { cians; } \\
\mathrm{n}=284,475 \\
\text { ratings) }\end{array}$ & $\begin{array}{l}2017 \\
(\mathrm{n}=79,799 \\
\text { rated } \\
\text { physi- } \\
\text { cians; } \\
\mathrm{n}=293,744 \\
\text { ratings) }\end{array}$ & $\begin{array}{l}2018 \\
(\mathrm{n}=82,551 \\
\text { rated } \\
\text { physi- } \\
\text { cians; } \\
\mathrm{n}=292,721 \\
\text { ratings) }\end{array}$ & $\begin{array}{l}2019 \\
(\mathrm{n}=73,071 \\
\text { rated } \\
\text { physi- } \\
\text { cians; } \\
\mathrm{n}=232,729 \\
\text { ratings })\end{array}$ & \\
\hline $\begin{array}{l}\text { Number of } \\
\text { ratings, } \mathrm{n} \\
(\%)\end{array}$ & $\begin{array}{l}1563 \\
(4.90)\end{array}$ & $\begin{array}{l}3199 \\
(5.18)\end{array}$ & $\begin{array}{l}5811 \\
(5.93)\end{array}$ & $\begin{array}{l}10,461 \\
(6.79)\end{array}$ & $\begin{array}{l}14,991 \\
(6.84)\end{array}$ & $\begin{array}{l}15,380 \\
(6.48)\end{array}$ & $\begin{array}{l}17,513 \\
(6.16)\end{array}$ & $\begin{array}{l}17,619 \\
(6.00)\end{array}$ & $\begin{array}{l}17,861 \\
(6.10)\end{array}$ & $\begin{array}{l}13,355 \\
(5.74)\end{array}$ & $\begin{array}{l}117,753 \\
(6.18)\end{array}$ \\
\hline $\begin{array}{l}\text { Mean rat- } \\
\text { ing }(\mathrm{SD})^{\mathrm{a}}\end{array}$ & $\begin{array}{l}2.06 \\
(1.64)\end{array}$ & $\begin{array}{l}2.18 \\
(1.71)\end{array}$ & $\begin{array}{l}2.35 \\
(1.85)\end{array}$ & $\begin{array}{l}2.28 \\
(1.82)\end{array}$ & $\begin{array}{l}2.25 \\
(1.82)\end{array}$ & $\begin{array}{l}2.16 \\
(1.77)\end{array}$ & $\begin{array}{l}2.05 \\
(1.73)\end{array}$ & $\begin{array}{l}2.04 \\
(1.74)\end{array}$ & $\begin{array}{l}2.04 \\
(1.75)\end{array}$ & $\begin{array}{l}1.94 \\
(1.71)\end{array}$ & $\begin{array}{l}2.11 \\
(1.77)\end{array}$ \\
\hline \multicolumn{12}{|c|}{ ENT $^{b}$ specialist, otorhinolaryngologist } \\
\hline $\begin{array}{l}\text { Rated } \\
\text { physicians, } \\
\mathrm{n}(\%)\end{array}$ & $\begin{array}{l}835 \\
(4.33)\end{array}$ & $\begin{array}{l}1388 \\
(4.43)\end{array}$ & $\begin{array}{l}1876 \\
(4.46)\end{array}$ & $\begin{array}{l}2425 \\
(4.57)\end{array}$ & $\begin{array}{l}2828 \\
(4.45)\end{array}$ & $\begin{array}{l}3094 \\
(4.52)\end{array}$ & $\begin{array}{l}3345 \\
(4.36)\end{array}$ & $\begin{array}{l}3443 \\
(4.31)\end{array}$ & $\begin{array}{l}3559 \\
(4.31)\end{array}$ & $\begin{array}{l}3233 \\
(4.42)\end{array}$ & $\begin{array}{l}4709 \\
(3.68)\end{array}$ \\
\hline $\begin{array}{l}\text { Number of } \\
\text { ratings, } \mathrm{n} \\
(\%)\end{array}$ & $\begin{array}{l}1455 \\
(4.56)\end{array}$ & $\begin{array}{l}3018 \\
(4.89)\end{array}$ & $\begin{array}{l}5081 \\
(5.18)\end{array}$ & $\begin{array}{l}9013 \\
(5.85)\end{array}$ & $\begin{array}{l}13,494 \\
(6.15)\end{array}$ & $\begin{array}{l}14,626 \\
(6.16)\end{array}$ & $\begin{array}{l}17,107 \\
(6.01)\end{array}$ & $\begin{array}{l}16,914 \\
(5.76)\end{array}$ & $\begin{array}{l}16,118 \\
(5.51)\end{array}$ & $\begin{array}{l}13,077 \\
(5.62)\end{array}$ & $\begin{array}{l}109,903 \\
(5.77)\end{array}$ \\
\hline $\begin{array}{l}\text { Mean rat- } \\
\text { ing }(S D)^{a}\end{array}$ & $\begin{array}{l}1.81 \\
(1.50)\end{array}$ & $\begin{array}{l}1.77 \\
(1.46)\end{array}$ & $\begin{array}{l}1.76 \\
(1.50)\end{array}$ & $\begin{array}{l}1.83 \\
(1.57)\end{array}$ & $\begin{array}{l}1.75 \\
(1.51)\end{array}$ & $\begin{array}{l}1.74 \\
(1.52)\end{array}$ & $\begin{array}{l}1.64 \\
(1.43)\end{array}$ & $\begin{array}{l}1.67 \\
(1.47)\end{array}$ & $\begin{array}{l}1.75 \\
(1.56)\end{array}$ & $\begin{array}{l}1.71 \\
(1.53)\end{array}$ & $\begin{array}{l}1.72 \\
(1.51)\end{array}$ \\
\hline $\begin{array}{l}\text { Rated } \\
\text { physicians, } \\
\text { n }(\%)\end{array}$ & $\begin{array}{l}601 \\
(3.11)\end{array}$ & $\begin{array}{l}1027 \\
(3.28)\end{array}$ & $\begin{array}{l}1397 \\
(3.32)\end{array}$ & $\begin{array}{l}1836 \\
(3.46)\end{array}$ & $\begin{array}{l}2150 \\
(3.40)\end{array}$ & $\begin{array}{l}2463 \\
(3.60)\end{array}$ & $\begin{array}{l}2791 \\
(3.64)\end{array}$ & $\begin{array}{l}3054 \\
(3.83)\end{array}$ & $\begin{array}{l}3154 \\
(3.82)\end{array}$ & $\begin{array}{l}2859 \\
(3.91)\end{array}$ & $\begin{array}{l}4343 \\
(3.40)\end{array}$ \\
\hline $\begin{array}{l}\text { Number of } \\
\text { ratings, } \mathrm{n} \\
(\%)\end{array}$ & $\begin{array}{l}1061 \\
(3.33)\end{array}$ & $\begin{array}{l}2298 \\
(3.72)\end{array}$ & $\begin{array}{l}3661 \\
(3.73)\end{array}$ & $\begin{array}{l}6103 \\
(3.96)\end{array}$ & $\begin{array}{l}9084 \\
(4.14)\end{array}$ & $\begin{array}{l}10,908 \\
(4.60)\end{array}$ & $\begin{array}{l}13,240 \\
(4.65)\end{array}$ & $\begin{array}{l}14,678 \\
(5.00)\end{array}$ & $\begin{array}{l}14,162 \\
(4.84)\end{array}$ & $\begin{array}{l}12,272 \\
(5.27)\end{array}$ & $\begin{array}{l}87,467 \\
(4.59)\end{array}$ \\
\hline $\begin{array}{l}\text { Mean rat- } \\
\text { ing }(S D)^{a}\end{array}$ & $\begin{array}{l}1.80 \\
(1.49)\end{array}$ & $\begin{array}{l}1.84 \\
(1.57)\end{array}$ & $\begin{array}{l}1.83 \\
(1.59)\end{array}$ & $\begin{array}{l}1.83 \\
(1.59)\end{array}$ & $\begin{array}{l}1.84 \\
(1.61)\end{array}$ & $\begin{array}{l}1.83 \\
(1.62)\end{array}$ & $\begin{array}{l}1.79 \\
(1.60)\end{array}$ & $\begin{array}{l}1.81 \\
(1.63)\end{array}$ & $\begin{array}{l}1.85 \\
(1.67)\end{array}$ & $\begin{array}{l}1.78 \\
(1.62)\end{array}$ & $\begin{array}{l}1.82 \\
(1.62)\end{array}$ \\
\hline \multicolumn{12}{|l|}{ Pediatrician } \\
\hline $\begin{array}{l}\text { Rated } \\
\text { physicians, } \\
\text { n }(\%)\end{array}$ & $\begin{array}{l}976 \\
(5.06)\end{array}$ & $\begin{array}{l}1570 \\
(5.01)\end{array}$ & $\begin{array}{l}2321 \\
(5.51)\end{array}$ & $\begin{array}{l}2996 \\
(5.65)\end{array}$ & $\begin{array}{l}3574 \\
(5.66)\end{array}$ & $\begin{array}{l}3891 \\
(5.69)\end{array}$ & $\begin{array}{l}4230 \\
(5.51)\end{array}$ & $\begin{array}{l}4315 \\
(5.41)\end{array}$ & $\begin{array}{l}4364 \\
(5.29)\end{array}$ & $\begin{array}{l}3620 \\
(4.95)\end{array}$ & $\begin{array}{l}6555 \\
(5.12)\end{array}$ \\
\hline $\begin{array}{l}\text { Number of } \\
\text { ratings, } \mathrm{n} \\
(\%)\end{array}$ & $\begin{array}{l}1529 \\
(4.81)\end{array}$ & $\begin{array}{l}2795 \\
(4.53)\end{array}$ & $\begin{array}{l}4941 \\
(5.04)\end{array}$ & $\begin{array}{l}7831 \\
(5.08)\end{array}$ & $\begin{array}{l}11,059 \\
(5.04)\end{array}$ & $\begin{array}{l}11,550 \\
(4.87)\end{array}$ & $\begin{array}{l}13,004 \\
(4.57)\end{array}$ & $\begin{array}{l}13,295 \\
(4.53)\end{array}$ & $\begin{array}{l}12,894 \\
(4.40)\end{array}$ & $\begin{array}{l}8432 \\
(3.62)\end{array}$ & $\begin{array}{l}87,330 \\
(4.58)\end{array}$ \\
\hline $\begin{array}{l}\text { Mean rat- } \\
\text { ing }(\mathrm{SD})^{\mathrm{a}}\end{array}$ & $\begin{array}{l}1.68 \\
(1.35)\end{array}$ & $\begin{array}{l}1.70 \\
(1.38)\end{array}$ & $\begin{array}{l}1.76 \\
(1.46)\end{array}$ & $\begin{array}{l}1.88 \\
(1.57)\end{array}$ & $\begin{array}{l}1.94 \\
(1.61)\end{array}$ & $\begin{array}{l}1.94 \\
(1.61)\end{array}$ & $\begin{array}{l}1.90 \\
(1.60)\end{array}$ & $\begin{array}{l}1.93 \\
(1.64)\end{array}$ & $\begin{array}{l}2.03 \\
(1.72)\end{array}$ & $\begin{array}{l}2.01 \\
(1.73)\end{array}$ & $\begin{array}{l}1.92 \\
(1.62)\end{array}$ \\
\hline \multicolumn{12}{|l|}{ Ophthalmologist } \\
\hline $\begin{array}{l}\text { Rated } \\
\text { physicians, } \\
\text { n }(\%)\end{array}$ & $\begin{array}{l}722 \\
(3.74)\end{array}$ & $\begin{array}{l}1225 \\
(3.91)\end{array}$ & $\begin{array}{l}1772 \\
(4.21)\end{array}$ & $\begin{array}{l}2366 \\
(4.46)\end{array}$ & $\begin{array}{l}2922 \\
(4.62)\end{array}$ & $\begin{array}{l}3131 \\
(4.58)\end{array}$ & $\begin{array}{l}3528 \\
(4.60)\end{array}$ & $\begin{array}{l}3809 \\
(4.77)\end{array}$ & $\begin{array}{l}3916 \\
(4.74)\end{array}$ & $\begin{array}{l}3520 \\
(4.82)\end{array}$ & $\begin{array}{l}5935 \\
(4.64)\end{array}$ \\
\hline $\begin{array}{l}\text { Number of } \\
\text { ratings, } \mathrm{n} \\
(\%)\end{array}$ & $\begin{array}{l}1085 \\
(3.40)\end{array}$ & $\begin{array}{l}2079 \\
(3.37)\end{array}$ & $\begin{array}{l}3570 \\
(3.64)\end{array}$ & $\begin{array}{l}6173 \\
(4.01)\end{array}$ & $\begin{array}{l}9154 \\
(4.17)\end{array}$ & $\begin{array}{l}9754 \\
(4.11)\end{array}$ & $\begin{array}{l}11,899 \\
(4.18)\end{array}$ & $\begin{array}{l}12,816 \\
(4.36)\end{array}$ & $\begin{array}{l}12,887 \\
(4.40)\end{array}$ & $\begin{array}{l}10,282 \\
(4.41)\end{array}$ & $\begin{array}{l}79,699 \\
(4.18)\end{array}$ \\
\hline
\end{tabular}




\begin{tabular}{|c|c|c|c|c|c|c|c|c|c|c|c|}
\hline \multirow{2}{*}{$\begin{array}{l}\text { Medical } \\
\text { specialty }\end{array}$} & \multicolumn{10}{|l|}{ Year } & \multirow{2}{*}{$\begin{array}{l}\text { Overall } \\
(\mathrm{n}=127,921 \\
\text { rated } \\
\text { physi- } \\
\text { cians; } \\
\mathrm{n}=1,906,146 \\
\text { ratings })\end{array}$} \\
\hline & $\begin{array}{l}2010 \\
(\mathrm{n}=19,305 \\
\text { rated } \\
\text { physi- } \\
\text { cians; } \\
\mathrm{n}=31,908 \\
\text { ratings })\end{array}$ & $\begin{array}{l}2011 \\
(\mathrm{n}=31,336 \\
\text { rated } \\
\text { physi- } \\
\text { cians; } \\
\mathrm{n}=61,726 \\
\text { ratings })\end{array}$ & $\begin{array}{l}2012 \\
(\mathrm{n}=42,089 \\
\text { rated } \\
\text { physi- } \\
\text { cians; } \\
\mathrm{n}=98,041 \\
\text { ratings })\end{array}$ & $\begin{array}{l}2013 \\
(\mathrm{n}=53,065 \\
\text { rated } \\
\text { physi- } \\
\text { cians; } \\
\mathrm{n}=154,119 \\
\text { ratings })\end{array}$ & $\begin{array}{l}2014 \\
(\mathrm{n}=63,182 \\
\text { rated } \\
\text { physi- } \\
\text { cians; } \\
\mathrm{n}=219,319 \\
\text { ratings) }\end{array}$ & $\begin{array}{l}2015 \\
(\mathrm{n}=68,392 \\
\text { rated } \\
\text { physi- } \\
\text { cians; } \\
\mathrm{n}=237,354 \\
\text { ratings) }\end{array}$ & $\begin{array}{l}2016 \\
(\mathrm{n}=76,773 \\
\text { rated } \\
\text { physi- } \\
\text { cians; } \\
\mathrm{n}=284,475 \\
\text { ratings })\end{array}$ & $\begin{array}{l}2017 \\
(\mathrm{n}=79,799 \\
\text { rated } \\
\text { physi- } \\
\text { cians; } \\
\mathrm{n}=293,744 \\
\text { ratings })\end{array}$ & $\begin{array}{l}2018 \\
(\mathrm{n}=82,551 \\
\text { rated } \\
\text { physi- } \\
\text { cians; } \\
\mathrm{n}=292,721 \\
\text { ratings })\end{array}$ & $\begin{array}{l}2019 \\
(\mathrm{n}=73,071 \\
\text { rated } \\
\text { physi- } \\
\text { cians; } \\
\mathrm{n}=232,729 \\
\text { ratings })\end{array}$ & \\
\hline \multicolumn{12}{|l|}{ Urologist } \\
\hline $\begin{array}{l}\text { Rated } \\
\text { physicians, } \\
\mathrm{n}(\%)\end{array}$ & $\begin{array}{l}536 \\
(2.78)\end{array}$ & $\begin{array}{l}830 \\
(2.65)\end{array}$ & $\begin{array}{l}1221 \\
(2.90)\end{array}$ & $\begin{array}{l}1511 \\
(2.85)\end{array}$ & $\begin{array}{l}1820 \\
(2.88)\end{array}$ & $\begin{array}{l}1914 \\
(2.80)\end{array}$ & $\begin{array}{l}2139 \\
(2.79)\end{array}$ & $\begin{array}{l}2301 \\
(2.88)\end{array}$ & $\begin{array}{l}2415 \\
(2.93)\end{array}$ & $\begin{array}{l}2140 \\
(2.93)\end{array}$ & $\begin{array}{l}3329 \\
(2.60)\end{array}$ \\
\hline $\begin{array}{l}\text { Number of } \\
\text { ratings, } \mathrm{n} \\
(\%)\end{array}$ & $\begin{array}{l}845 \\
(2.65)\end{array}$ & $\begin{array}{l}1639 \\
(2.66)\end{array}$ & $\begin{array}{l}3221 \\
(3.29)\end{array}$ & $\begin{array}{l}5141 \\
(3.34)\end{array}$ & $\begin{array}{l}7207 \\
(3.29)\end{array}$ & $\begin{array}{l}7753 \\
(3.27)\end{array}$ & $\begin{array}{l}9556 \\
(3.36)\end{array}$ & $\begin{array}{l}10,264 \\
(3.49)\end{array}$ & $\begin{array}{l}9612 \\
(3.28)\end{array}$ & $\begin{array}{l}8465 \\
(3.64)\end{array}$ & $\begin{array}{l}63,703 \\
(3.34)\end{array}$ \\
\hline $\begin{array}{l}\text { Mean rat- } \\
\text { ing }(\mathrm{SD})^{\mathrm{a}}\end{array}$ & $\begin{array}{l}1.82 \\
(1.54)\end{array}$ & $\begin{array}{l}1.66 \\
(1.37)\end{array}$ & $\begin{array}{l}1.57 \\
(1.33)\end{array}$ & $\begin{array}{l}1.64 \\
(1.41)\end{array}$ & $\begin{array}{l}1.54 \\
(1.30)\end{array}$ & $\begin{array}{l}1.50 \\
(1.28)\end{array}$ & $\begin{array}{l}1.43 \\
(1.20)\end{array}$ & $\begin{array}{l}1.47 \\
(1.26)\end{array}$ & $\begin{array}{l}1.49 \\
(1.29)\end{array}$ & $\begin{array}{l}1.45 \\
(1.25)\end{array}$ & $\begin{array}{l}1.50 \\
(1.29)\end{array}$ \\
\hline \multicolumn{12}{|l|}{ Others } \\
\hline $\begin{array}{l}\text { Rated } \\
\text { physicians, } \\
\mathrm{n}(\%)\end{array}$ & $\begin{array}{l}2840 \\
(14.71)\end{array}$ & $\begin{array}{l}4379 \\
(13.97)\end{array}$ & $\begin{array}{l}5708 \\
(13.56)\end{array}$ & $\begin{array}{l}7310 \\
(13.78)\end{array}$ & $\begin{array}{l}8883 \\
(14.06)\end{array}$ & $\begin{array}{l}9716 \\
(14.21)\end{array}$ & $\begin{array}{l}11,104 \\
(14.46)\end{array}$ & $\begin{array}{l}11,675 \\
(14.63)\end{array}$ & $\begin{array}{l}12,127 \\
(14.69)\end{array}$ & $\begin{array}{l}10,799 \\
(14.78)\end{array}$ & $\begin{array}{l}21,765 \\
(17.01)\end{array}$ \\
\hline $\begin{array}{l}\text { Number of } \\
\text { ratings, } \mathrm{n} \\
(\%)\end{array}$ & $\begin{array}{l}4684 \\
(14.68)\end{array}$ & $\begin{array}{l}8944 \\
(14.49)\end{array}$ & $\begin{array}{l}13,842 \\
(14.12)\end{array}$ & $\begin{array}{l}20,907 \\
(13.57)\end{array}$ & $\begin{array}{l}30,302 \\
(13.82)\end{array}$ & $\begin{array}{l}34,913 \\
(14.71)\end{array}$ & $\begin{array}{l}42,929 \\
(15.09)\end{array}$ & $\begin{array}{l}44,871 \\
(15.28)\end{array}$ & $\begin{array}{l}46,737 \\
(15.97)\end{array}$ & $\begin{array}{l}39,626 \\
(17.03)\end{array}$ & $\begin{array}{l}287,755 \\
(15.10)\end{array}$ \\
\hline $\begin{array}{l}\text { Mean rat- } \\
\text { ing }(\mathrm{SD})^{\mathrm{a}}\end{array}$ & $\begin{array}{l}1.78 \\
(1.48)\end{array}$ & $\begin{array}{l}1.77 \\
(1.49)\end{array}$ & $\begin{array}{l}1.68 \\
(1.45)\end{array}$ & $\begin{array}{l}1.76 \\
(1.54)\end{array}$ & $\begin{array}{l}1.70 \\
(1.49)\end{array}$ & $\begin{array}{l}1.65 \\
(1.45)\end{array}$ & $\begin{array}{l}1.59 \\
(1.40)\end{array}$ & $\begin{array}{l}1.62 \\
(1.43)\end{array}$ & $\begin{array}{l}1.66 \\
(1.49)\end{array}$ & $\begin{array}{l}1.57 \\
(1.41)\end{array}$ & $\begin{array}{l}1.65 \\
(1.45)\end{array}$ \\
\hline
\end{tabular}

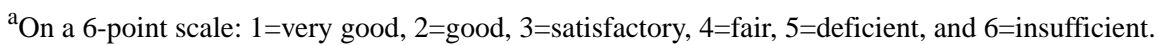

${ }^{\mathrm{b}} \mathrm{ENT}$ : ear, nose, throat.

\section{Characteristics of Raters}

The rating patients were mostly female $(56.8 \%)$, between 30 and 50 years old $(42.6 \%)$, and covered by Statutory Health Insurance $(81.0 \%$; see Table 6). However, there were some significant differences between genders, age groups, and health insurance status. Male patients gave significantly more favorable ratings than female patients (mean rating 1.61, SD 1.32 vs. mean 1.77, SD 1.48; $P<.001$ ). Older patients also gave significantly better ratings than younger patients $(P<.001)$. For example, patients aged 51 years or older left a mean rating of 1.52 (SD 1.22), whereas patients aged 29 years or younger left a mean rating of 1.93 (SD 1.59). Finally, patients covered by private health insurance (mean rating 1.43 , SD 1.11) gave significantly more favorable evaluations than did patients covered by statutory health insurance (mean rating 1.75, SD 1.47; $P<.001$ ). Nevertheless, effect sizes were small for all groups, varying between 0.114 and 0.289 . 
Table 6. Characteristics of raters.

\begin{tabular}{|c|c|c|c|c|}
\hline Characteristic & Number of respondents, $\mathrm{n}(\%)$ & Rating evaluation, mean (SD) & $P$ value & Cohen $d$ \\
\hline \multicolumn{5}{|l|}{ Gender $(n=1,107,092)$} \\
\hline Male & $478,592(43.23)$ & $1.61(1.32)$ & $<.001^{\mathrm{a}}$ & 0.114 \\
\hline Female & $628,500(56.77)$ & $1.77(1.48)$ & & \\
\hline \multicolumn{5}{|l|}{ Age (years; n=1,063,523) } \\
\hline$\leq 29$ & $164,807(15.50)$ & $1.93(1.59)$ & $<.001^{\mathrm{b}}$ & $0.117^{\mathrm{c}} ; 0.289^{\mathrm{d}}$ \\
\hline $30-50$ & $452,774(42.57)$ & $1.75(1.46)$ & & $0.171^{\mathrm{e}}$ \\
\hline$\geq 51$ & $445,942(41.93)$ & $1.52(1.22)$ & & \\
\hline \multicolumn{5}{|l|}{ Health insurance $(\mathbf{n}=981,635)$} \\
\hline Statutory health insurance & $795,107(81.00)$ & $1.75(1.47)$ & $<.001^{\mathrm{a}}$ & 0.245 \\
\hline Private health insurance & $186,528(19.00)$ & $1.43(1.11)$ & & \\
\hline
\end{tabular}

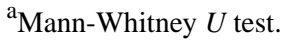

${ }^{\mathrm{b}}$ Kruskal-Wallis test.

$\mathrm{c} \leq 29$ years vs $30-50$ years.

$\mathrm{d}_{\leq 29}$ years vs $\geq 51$ or years.

$\mathrm{e}_{30-50 \text { years } v s} \geq 51$ years.

\section{Discussion}

This study is one of the most comprehensive analyses of PRW ratings conducted to date and has resulted in a number of key findings: (1) just under 2 million ratings were posted on jameda between 2010 and 2019; (2) a total of 127,921 physicians were rated; (3) the overall average number of ratings per rated physicians nearly doubled; (4) three-quarters of all ratings were in the best rating category of "very good," and the overall average rating remained relatively constant; (5) general practitioners, internists, gynecologists, and orthopedists were the most frequently rated medical specialties; and (6) the rating patients were mostly female, between 30 and 50 years old, and covered by Statutory Health Insurance.

The findings of this study confirm previous research in Germany that indicated that patient ratings show an increasing trend over the past decade [26]. For example, the percentage of all German physicians that had been rated on jameda increased constantly over time from $13.65 \%(19,305 / 141,461)$ in 2010 to $52.46 \%$ $(82,511 / 157,288)$ in 2018. McLennan et al [26] also previously reported that the proportion of physicians from a sample of 298 randomly selected physicians from Hamburg and Thuringia that had been rated at least once had increased between 2010 (range $3.3 \%-27.8 \%$ ) and 2014 (range 16.4\%-83.2\%). Similarly, the average number of ratings per physician also increased between 2010 (range 1.1-3.1) and 2014 (range 1.2-7.5). However, this study only used a small sample from 2 regions in Germany. Overall, there is little international evidence showing the exact development of online ratings over time, which makes it challenging to compare our numbers with those from other similar studies. To the best of our knowledge, more recent studies providing detailed information on a yearly basis are limited. However, 2 studies from the United States [13] and Canada [27] have presented similar findings. First, in 2012, Gao and colleagues [13] showed an increase in the number of rated physicians on RateMDs in the United States from 2475 in 2005 to 112,024 in 2010. Second, Liu and colleagues [27] analyzed a dataset from RateMDs, which included all physicians in Canada in 2018 and showed an increase in the number of ratings for physicians in Canada from 138 in 2005 to 640,603 in 2013. Nevertheless, it should be noted that this study found a plateau in the total number of ratings between $2017(293,744)$ and 2018 $(292,721)$. In 2019, a decrease of around $20 \%$ in the total number of ratings was seen in comparison with the previous 2 years. In recent years, jameda has implemented and promoted new features on its website (eg, making appointments, video consultations). This has possibly led to lower marketing efforts for collecting online reviews and may also lead to differences from PRWs not offering these addition services. Future studies should investigate whether this latest development can also be observed for other PRWs in Germany and other countries.

This study only provides information regarding jameda. Previous research has demonstrated much lower numbers of both ratings and rated physicians on other German PRWs [4,26]. For example, McLennan and colleagues [26] reported that between $16.4 \%$ and $71.1 \%$ (mean $41.4 \%$ ) of physicians were rated on German PRWs overall, compared with $83.2 \%$ on jameda. Another study also showed a higher percentage of rated physicians on jameda (90.2\%) compared with other relevant German PRWs (32.4\% to 61.2\%) [4]. Differences in the number of ratings between PRWs can also be shown in the international setting. For example, Trehan and colleagues [44] analyzed online ratings for 250 hand surgeons from the American Society for Surgery of the Hand member directory from 3 PRWs in the United States (HealthGrades, Vitals, RateMDs). Large differences were reported regarding the average number of ratings (13.4, 8.3, and 1.9, respectively) [44]. Further research is required to confirm that this increase in ratings is also true for other PRWs as well.

Furthermore, the percentages of ratings on both ends of the rating scale have increased. This may suggest that a "bimodal" trend in ratings is emerging on jameda, similar to that seen with 
the rating of products on websites like Amazon where "amateur" reviewers usually only leave a review because they either love or hate a product [45]. It would be helpful if future research examines if this trend continues and can be found on other PRWs, particularly as this trend is usually not seen on PRWs [26], despite qualitative research in Germany finding that a very positive or very negative experience in the health care relationship is a crucial precondition for patients to be willing to rate a physician [46].

Seven years after the first study on online patient ratings on jameda [18], general practitioners, internists, and gynecologists still receive the highest number of ratings in absolute terms. This does not seem surprising due to the high number of physicians in those medical specialty areas in Germany. Similar to previous research [18], it could also be shown that urologists, general practitioners, and internists were likely to receive more favorable ratings on jameda. In contrast, ophthalmologists and dermatologists are still likely to receive far less favorable ratings. This is also in line with the comprehensive analysis by Liu and colleagues [27] from Canada. Previous research findings have also reported that generalists are more likely to have better online ratings than specialists [10,13]. Qualitative research conducted in Germany by McLennan et al [46] found that factors concerning the physician-patient relationship to be some of the most important influencing people's willingness to rate their physician on PRWs. It is likely that differences in patients' relationships with physicians in various specialties (eg, duration and frequency of contact and the resulting level of trust) is a key factor for this heterogeneity.

The analysis of such a large number of ratings has also provided a more detailed picture of the association between the number of ratings a physician has and their overall evaluation. Although physicians with only 1 rating tended to have very good ratings ( $81 \%$ of all ratings were in the best rating category), this might potentially be explained, at least in part, by "fake ratings" left by physicians themselves or people connected to the physician. Regardless, it certainly calls into question whether results based on a single rating are meaningful at all [7]. Afterwards, more critical rating results were found. In line with previous studies from Germany [18] and the United States [37], the total performance range was found for physicians with a lower number of ratings. This possibly represents a more realistic picture of patient feedback because the percentage of ratings in the very best rating category declined constantly, and it is also likely that those physicians are not using PRWs as a marketing measure to collect a very high number or ratings [18]. However, in contrast to previous research, physicians who received a higher number of ratings were shown to have better ratings. When there were more than 51 ratings, ratings started to improve again, and physicians with more than 100 ratings received by far the most favorable ratings. It is likely that physician with more than 100 ratings are aware of PRWs and are using them as a marketing tool, potentially specifically asking satisfied patients to leave a (positive) rating on a PRW. However, it is possible that these physicians are simply providing outstanding quality of care, leading to the very favorable ratings on PRWs and, subsequently, more patients choosing to use this physician
[18]. Future research should examine which assumption is true [18].

In 2019, Pike et al [37] reported a U-shaped relationship between the number of ratings and the overall rating from the Healthgrades website. A negative relationship between the number of ratings and the overall rating could be seen until physicians achieved 21 ratings; thereafter, a positive relationship was seen. It should be noted that, in contrast to jameda, a lower score on Healthgrades means a worse rating ( $1=$ poor; $5=$ excellent). Although regression analysis on the jameda data did not find a satisfying fit, the study provides further broad-scale evidence on the relationship between the number of ratings and the overall evaluation as discussed earlier in this manuscript.

\section{Limitations}

The key limitation of this study is that it analyzed online ratings from only a single German PRW, jameda. Although jameda has shown to be the most frequently used German PRW, there are a total of 25 PRWs in Germany [8], and it is unclear how generalizable the results are to other German PRWs or to other countries. In Germany, it would be particularly helpful for future longitudinal research to examine trends in ratings on PRWs run by public health insurers, as previous research has indicated that these PRWs have been able to quickly establish themselves as some of the most used German PRWs alongside jameda [26]. Another limitation of the study is that it only analyzed publicly available ratings; it is not known how many additional ratings jameda received but did not publish or what efforts jameda made to check whether published ratings are genuine and not fake. Indeed, jameda has often been criticized with regards to the number of fake reviews and its business model that offers physicians paid premium profiles. Recent research has raised concerns that online patient feedback is being inappropriately manipulated by many PRWs and that business models that make PRWs reliant on paying physicians may create financial incentives to suppress negative feedback [47]. Although further work is needed on criteria for determining which feedback is published [47], it is also important to have a comprehensive understanding of the ratings that are being viewed by the public on PRWs.

\section{Conclusion}

In conclusion, it can be stated that online ratings have been increasing tremendously over the past decade and seem to have become an essential element for patients to leave feedback on the care they receive. More than half of all physicians have been rated online on jameda each year in Germany since 2016. Indeed, with patients increasingly using the internet in relation to their health care [48], it is likely that online patient feedback will become even more important in the future. With online patient feedback mostly positive, physicians do not have to fear online ratings in general; the commonly expressed concerns regarding PRWs being used for "doctorbashing" or defamation [31] or as "platforms for denunciation" [32] have not proven true. Furthermore, less favorable patient ratings often address important elements of a patient-oriented health care system [1] and can help organizations and professionals identify areas that need to be improved [21]. 


\section{Acknowledgments}

This publication was funded by the German Research Foundation (DFG) and the University of Bayreuth through the funding program Open Access Publishing.

The authors would like to thank Ms Elke Ruppert and the management from jameda for the provision of the data.

\section{Conflicts of Interest}

None declared.

\section{Multimedia Appendix 1}

The number and percentage of rated physicians in Germany.

[PNG File, 127 KB-Multimedia Appendix 1]

\section{Multimedia Appendix 2}

Number and distribution of ratings according to the medical specialty (2018).

[DOCX File, 37 KB-Multimedia Appendix 2]

\section{References}

1. Powell J, Atherton H, Williams V, Mazanderani F, Dudhwala F, Woolgar S, et al. Using online patient feedback to improve NHS services: the INQUIRE multimethod study. Health Serv Deliv Res 2019 Oct;7(38):1-150. [doi: 10.3310/hsdr07380] [Medline: 31693328$]$

2. Faber M, Bosch M, Wollersheim H, Leatherman S, Grol R. Public reporting in health care: how do consumers use quality-of-care information? A systematic review. Med Care 2009 Jan;47(1):1-8. [doi: 10.1097/MLR.0b013e3181808bb5] [Medline: 19106724]

3. Picker Institute. Using Patient Feedback. National Health Service (NHS) UK. 2009. URL: http://www.nhssurveys.org/ Filestore/documents/QIFull.pdf [accessed 2021-07-19]

4. Emmert M, Meszmer N. [A Decade of Online Physician-Rating Websites in Germany: an Assessment of the Current Level of Development]. Gesundheitswesen 2018 Oct;80(10):851-858 [FREE Full text] [doi: 10.1055/s-0043-114002] [Medline: 28759920]

5. Emmert M, Schlesinger M. Patients' Awareness, Usage and Impact of Hospital Report Cards in the US. Patient 2017 Dec;10(6):729-738 [FREE Full text] [doi: 10.1007/s40271-017-0243-y] [Medline: 28447273]

6. Emmert M, Becker S, Meszmer N, Sander U. [Are Facebook Ratings Associated with Hospital Quality and Patient Satisfaction? A Cross-Sectional Analysis of Obstetrics Care in German Hospitals]. Gesundheitswesen 2020 Jun;82(6):541-547 [FREE Full text] [doi: 10.1055/a-0774-7874] [Medline: 30477038]

7. Emmert M, Sander U, Pisch F. Eight questions about physician-rating websites: a systematic review. J Med Internet Res 2013 Feb 01;15(2):e24 [FREE Full text] [doi: 10.2196/jmir.2360] [Medline: 23372115]

8. Rothenfluh F, Schulz PJ. Content, Quality, and Assessment Tools of Physician-Rating Websites in 12 Countries: Quantitative Analysis. J Med Internet Res 2018 Jun 14;20(6):e212 [FREE Full text] [doi: 10.2196/jmir.9105] [Medline: 29903704]

9. Black E, Thompson L, Saliba H, Dawson K, Black NMP. An analysis of healthcare providers' online ratings. Inform Prim Care 2009 Dec 01;17(4):249-253 [FREE Full text] [doi: 10.14236/jhi.v17i4.744] [Medline: 20359403]

10. Lagu T, Hannon NS, Rothberg MB, Lindenauer PK. Patients' evaluations of health care providers in the era of social networking: an analysis of physician-rating websites. J Gen Intern Med 2010 Sep;25(9):942-946 [FREE Full text] [doi: 10.1007/s11606-010-1383-0] [Medline: 20464523]

11. Kadry B, Chu LF, Kadry B, Gammas D, Macario A. Analysis of 4999 online physician ratings indicates that most patients give physicians a favorable rating. J Med Internet Res 2011 Nov 16;13(4):e95 [FREE Full text] [doi: 10.2196/jmir.1960] [Medline: 22088924]

12. López A, Detz A, Ratanawongsa N, Sarkar U. What patients say about their doctors online: a qualitative content analysis. J Gen Intern Med 2012 Jun 4;27(6):685-692 [FREE Full text] [doi: 10.1007/s11606-011-1958-4] [Medline: 22215270]

13. Gao GG, McCullough JS, Agarwal R, Jha AK. A changing landscape of physician quality reporting: analysis of patients' online ratings of their physicians over a 5-year period. J Med Internet Res 2012 Feb 24;14(1):e38 [FREE Full text] [doi: 10.2196/jmir.2003] [Medline: 22366336]

14. Strech D, Reimann S. [German language physician rating sites]. Gesundheitswesen 2012 Aug; 74(8-9):e61-e67 [FREE Full text] [doi: 10.1055/s-0031-1297254] [Medline: 22189671]

15. Greaves F, Pape UJ, Lee H, Smith DM, Darzi A, Majeed A, et al. Patients' ratings of family physician practices on the internet: usage and associations with conventional measures of quality in the English National Health Service. J Med Internet Res 2012 Oct 17;14(5):e146 [FREE Full text] [doi: 10.2196/jmir.2280] [Medline: 23076301] 
16. Lagu T, Goff SL, Hannon NS, Shatz A, Lindenauer PK. A Mixed-Methods Analysis of Patient Reviews of Hospital Care in England: Implications for Public Reporting of Health Care Quality Data in the United States. The Joint Commission Journal on Quality and Patient Safety 2013 Jan;39(1):7-AP4. [doi: 10.1016/s1553-7250(13)39003-5]

17. Emmert M, Meier F, Pisch F, Sander U. Physician choice making and characteristics associated with using physician-rating websites: cross-sectional study. J Med Internet Res 2013 Aug 28;15(8):e187 [FREE Full text] [doi: 10.2196/jmir.2702] [Medline: 23985220]

18. Emmert M, Meier F. An analysis of online evaluations on a physician rating website: evidence from a German public reporting instrument. J Med Internet Res 2013 Aug 06;15(8):e157 [FREE Full text] [doi: 10.2196/jmir.2655] [Medline: 23919987]

19. Ellimoottil C, Hart A, Greco K, Quek ML, Farooq A. Online reviews of 500 urologists. J Urol 2013 Jun;189(6):2269-2273 [FREE Full text] [doi: 10.1016/j.juro.2012.12.013] [Medline: 23228385]

20. Emmert M, Gerstner B, Sander U, Wambach V. Eine Bestandsaufnahme von Bewertungen auf Arztbewertungsportalen am Beispiel des Nürnberger Gesundheitsnetzes Qualität und Effizienz (QuE). Gesundh ökon Qual manag 2013 Jul 9;19(04):161-167. [doi: 10.1055/s-0033-1335899]

21. Emmert M, Meier F, Heider AK, Dürr C, Sander U. What do patients say about their physicians? an analysis of 3000 narrative comments posted on a German physician rating website. Health Policy 2014 Oct;118(1):66-73 [FREE Full text] [doi: 10.1016/j.healthpol.2014.04.015] [Medline: 24836021]

22. Sobin L, Goyal P. Trends of online ratings of otolaryngologists: what do your patients really think of you? JAMA Otolaryngol Head Neck Surg 2014 Jul;140(7):635-638 [FREE Full text] [doi: 10.1001/jamaoto.2014.818] [Medline: 24876073]

23. Emmert M, Halling F, Meier F. Evaluations of dentists on a German physician rating Website: an analysis of the ratings. J Med Internet Res 2015 Jan 12;17(1):e15 [FREE Full text] [doi: 10.2196/jmir.3830] [Medline: 25582914]

24. Emmert M, Sauter L, Jablonski L, Sander U, Taheri-Zadeh F. Do Physicians Respond to Web-Based Patient Ratings? An Analysis of Physicians' Responses to More Than One Million Web-Based Ratings Over a Six-Year Period. J Med Internet Res 2017 Jul 26;19(7):e275 [FREE Full text] [doi: 10.2196/jmir.7538] [Medline: 28747292]

25. Murphy GP, Awad MA, Osterberg EC, Gaither TW, Chumnarnsongkhroh T, Washington SL, et al. Web-Based Physician Ratings for California Physicians on Probation. J Med Internet Res 2017 Aug 22;19(8):e254 [FREE Full text] [doi: 10.2196/jmir.7488] [Medline: 28830852]

26. McLennan S, Strech D, Reimann S. Developments in the Frequency of Ratings and Evaluation Tendencies: A Review of German Physician Rating Websites. J Med Internet Res 2017 Aug 25;19(8):e299 [FREE Full text] [doi: 10.2196/jmir.6599] [Medline: 28842391]

27. Liu JJ, Matelski JJ, Bell CM. Scope, Breadth, and Differences in Online Physician Ratings Related to Geography, Specialty, and Year: Observational Retrospective Study. J Med Internet Res 2018 Mar 07;20(3):e76 [FREE Full text] [doi: 10.2196/jmir.7475] [Medline: 29514775]

28. McLennan S. Quantitative Ratings and Narrative Comments on Swiss Physician Rating Websites: Frequency Analysis. J Med Internet Res 2019 Jul 26;21(7):e13816 [FREE Full text] [doi: 10.2196/13816] [Medline: 31350838]

29. McLennan S. The Content and Nature of Narrative Comments on Swiss Physician Rating Websites: Analysis of 849 Comments. J Med Internet Res 2019 Sep 30;21(9):e14336 [FREE Full text] [doi: 10.2196/14336] [Medline: 31573918]

30. Emmert M, Sander U, Kolb B, Meszmer N. Arztbewertungsportale: Die Kritik der Ärzte. Dtsch Arztebl 2017;114(15):731.

31. Carvel J. Patients to rate and review their GPs on NHS website: Doctors say bulletin board will be 'meaningless popularity contest'. The Guardian. 2008 Dec 29. URL: https://www.theguardian.com/society/2008/dec/30/doctors-rating-website-nhs [accessed 2021-07-19]

32. Arztbewertungsportale: Massie Kritik am AOK-Arzt-Navigator: BÄK Newsletter 2009-004, 2009. Ärztlicher Kreisverband Ebersberg. 2009 Jun 25. URL: https://www.aekv-ebersberg.de/aktuelles/105-baek-newsletter-2009-004-vom-25062009 [accessed 2021-07-19]

33. Bundesgerichtshof lehnt den Anspruch eines Arztes auf Löschung seiner Daten aus einem Ärztebewertungsportal. Bundesgerichtshof. 2014. URL: http://juris.bundesgerichtshof.de/cgi-bin/rechtsprechung/document. py?Gericht=bgh\&Art=en \&sid=18e188ccd712bed4e8781314de1c4c63\&anz=1\&pos=0\&nr=68915\&linked=pm\&Blank=1 [accessed 2021-07-19]

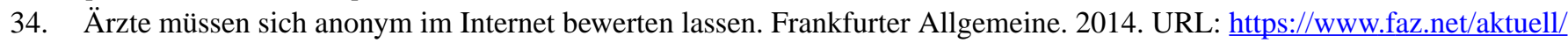
finanzen/meine-finanzen/geld-ausgeben/nachrichten/aerzteportale-jameda-urteil-az-bgh-vi-zr-358-13-13169241.html [accessed 2021-07-19]

35. Emmert M, Meszmer N, Sander U. Do Health Care Providers Use Online Patient Ratings to Improve the Quality of Care? Results From an Online-Based Cross-Sectional Study. J Med Internet Res 2016 Sep 19;18(9):e254 [FREE Full text] [doi: 10.2196/jmir.5889] [Medline: 27644135]

36. Meszmer N, Jaegers L, Schöffski O, Emmert M. [Do online ratings reflect structural differences in healthcare? The example of German physician-rating websites]. Z Evid Fortbild Qual Gesundhwes 2018 Apr;131-132:73-80 [FREE Full text] [doi: 10.1016/j.zefq.2017.11.007] [Medline: 29331281]

37. Pike CW, Zillioux J, Rapp D. Online Ratings of Urologists: Comprehensive Analysis. J Med Internet Res 2019 Jul 02;21(7):e12436 [FREE Full text] [doi: 10.2196/12436] [Medline: 31267982] 
38. Bakhsh W, Mesfin A. Online ratings of orthopedic surgeons: analysis of 2185 reviews. Am J Orthop (Belle Mead NJ) 2014 Aug;43(8):359-363. [Medline: 25136868]

39. Bovenzi CD, Manges KA, Krein H, Heffelfinger R. Online Ratings of Facial Plastic Surgeons: Worthwhile Additions to Conventional Patient Experience Surveys. Facial Plast Surg Aesthet Med 2021;23(2):78-89 [FREE Full text] [doi: 10.1089/fpsam.2020.0049] [Medline: $\underline{\text { 32716653] }}$

40. Cloney M, Hopkins B, Shlobin N, Dahdaleh NS. Online Ratings of Neurosurgeons: An Examination of Web Data and its Implications. Neurosurgery 2018 Dec 01;83(6):1143-1152 [FREE Full text] [doi: 10.1093/neuros/nyy064] [Medline: 29618127]

41. Haffey SC, Hopman WM, Leveridge MJ. Physicians' Earnings Do Not Affect Their Online Ratings. Front Public Health 2020;8:300 [FREE Full text] [doi: 10.3389/fpubh.2020.00300] [Medline: 32733838]

42. Jameda. URL: https://www.jameda.de/ [accessed 2021-07-17]

43. Die ärztliche Versorgung in Deutschland. Bundesaerztekammer. 2020. URL: https://www.bundesaerztekammer.de/ueber-uns/ aerztestatistik/ [accessed 2021-07-19]

44. Trehan SK, DeFrancesco CJ, Nguyen JT, Charalel RA, Daluiski A. Online Patient Ratings of Hand Surgeons. J Hand Surg Am 2016 Jan;41(1):98-103 [FREE Full text] [doi: 10.1016/j.jhsa.2015.10.006] [Medline: 26710742]

45. Gilbert E, Karahalios K. Understanding deja reviewers. 2010 Presented at: ACM conference on Computer supported cooperative work; February 6-10, 2010; Savannah, GA URL: https://doi.org/10.1145/1718918.1718961 [doi: 10.1145/1718918.1718961]

46. McLennan S, Strech D, Kahrass H. Why are so few patients rating their physicians on German physician rating websites? A qualitative study. BMC Health Serv Res 2018 Aug 29;18(1):670 [FREE Full text] [doi: 10.1186/s12913-018-3492-0] [Medline: 30157842$]$

47. McLennan S. Rejected Online Feedback From a Swiss Physician Rating Website Between 2008 and 2017: Analysis of 2352 Ratings. J Med Internet Res 2020 Aug 03;22(8):e18374 [FREE Full text] [doi: 10.2196/18374] [Medline: 32687479]

48. Eysenbach G. The impact of the Internet on cancer outcomes. CA Cancer J Clin 2003;53(6):356-371 [FREE Full text] [doi: 10.3322/canjclin.53.6.356] [Medline: 15224975]

\section{Abbreviations \\ ENT: ear, nose, and throat \\ PRW: physician rating website}

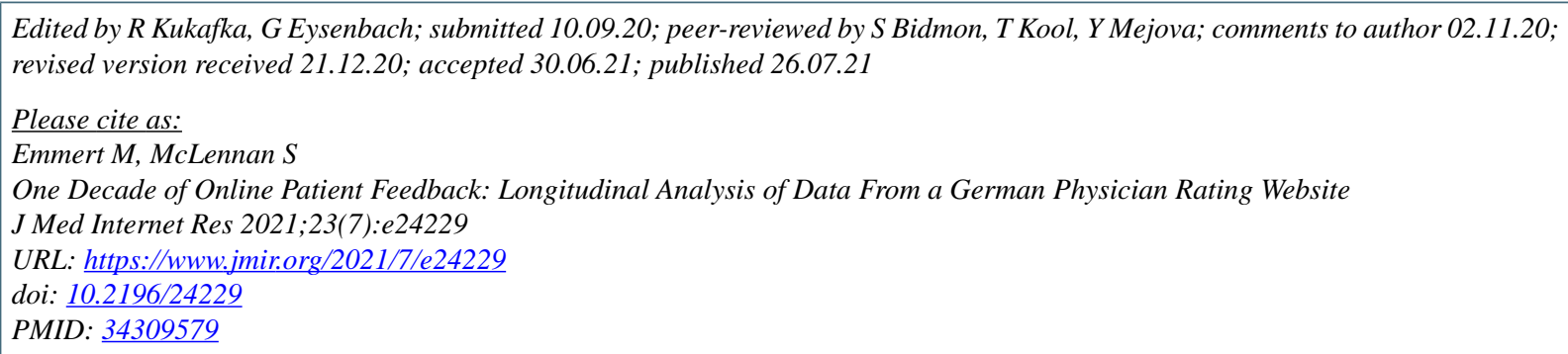

CMartin Emmert, Stuart McLennan. Originally published in the Journal of Medical Internet Research (https://www.jmir.org), 26.07.2021. This is an open-access article distributed under the terms of the Creative Commons Attribution License (https://creativecommons.org/licenses/by/4.0/), which permits unrestricted use, distribution, and reproduction in any medium, provided the original work, first published in the Journal of Medical Internet Research, is properly cited. The complete bibliographic information, a link to the original publication on https://www.jmir.org/, as well as this copyright and license information must be included. 